\title{
SIMPLE TRANSITIVE 2-REPRESENTATIONS FOR TWO NON-FIAT 2-CATEGORIES OF PROJECTIVE FUNCTORS
}

\author{
VOLODYMYR MAZORCHUK AND XIAOTING ZHANG
}

\begin{abstract}
We show that any simple transitive 2-representation of the 2-category of projective endofunctors for the quiver algebra of $\mathbb{k}(\bullet \longrightarrow \bullet)$ and for the quiver algebra of $\mathbb{k}(\bullet \stackrel{\cdots \cdots \cdots}{\longrightarrow} \bullet)$ is equivalent to a cell 2-representation.
\end{abstract}

\section{INTRODUCTION AND DESCRIPTION OF THE RESULTS}

Classification problems are interesting and important problems in the classical representation theory. For example, classifications of various classes of simple or indecomposable modules over different classes of algebras played significant role in both development and applications of modern representation theory.

Higher representation theory is a recent direction of representation theory that takes its origins from the papers BFK, CR, Ro1, Ro2. Of particular interest in higher representation theory is the study of so-called finitary 2-categories as the latter are natural 2-analogues of finite dimensional algebras. Initial abstract study of finitary 2-categories and the corresponding 2-representation theory was done in MM1, MM2, MM3, MM4, MM5, MM6, Xa].

As an outcome of this study, one interesting and important class of 2-representations, called simple transitive 2-representations, was defined in MM5. These 2-representations are natural 2-analogues of usual simple modules over algebras. Therefore the problem of classification of simple transitive 2-representations is natural and interesting. In several cases, it turns out that simple transitive 2-representations can be classified, see for example various results in MM5, MM6, GM1, Zh2, Zi . We also refer the reader to GM2, KiMa, Maz, Zh1 to related questions and applications. In particular, in [KiMa, classification of simple transitive 2-representations for the 2-category of Soergel bimodules over the coinvariant algebra of the symmetric group was crucially used for classification of projective functors in parabolic category $\mathcal{O}$ for $\mathfrak{s l}_{n}$.

The most basic example of a 2-category is the 2-category $\mathscr{C}_{A}$ of projective functors for a finite-dimensional algebra $A$ over an algebraically closed field $\mathbb{k}$, defined in MM1, Subsection 7.3]. In [MM3, MM6, it is shown that categories of the form $\mathscr{C}_{A}$ essentially exhausts a natural class of "simple" finitary 2-categories possessing weak involutions. For such 2-categories, it was shown in MM5, MM6 that simple transitive 2-representations are exactly the cell 2-representations, defined in [MM1. Existence of a weak involution on a 2-category restricts the classification result to the case when $A$ is a self-injective algebra.

The aim of the present paper to classify simple transitive 2-representations of $\mathscr{C}_{A}$ for the smallest possible non-self-injective algebra, namely the path algebra $A$ of the quiver $1 \longrightarrow 2$, over an algebraically closed field $\mathbb{k}$. It turns out that our approach also extends, with a significantly increased amount of technical work, to the quiver 
algebra of $\mathbb{k}(\bullet \stackrel{\cdots \cdots \cdots \cdots}{\longrightarrow} \bullet \bullet)$, where, as usual, the dotted arrow depicts the corresponding zero relation. Our main result is the following theorem, we refer the reader to Sections 2 for details on all definitions.

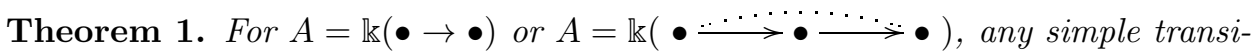
tive 2-representation of the 2-category $\mathscr{C}_{A}$ is equivalent to a cell 2-representation.

Despite of the fact that the formulation of Theorem 1 is rather similar to the corresponding statement in the case when $A$ is self-injective, considered in MM5, MM6, our approach to the proof is fairly different, since the general approach outlined in MM5, MM6 does not apply. Our approach, rather, has many similarities with the approach in $\mathrm{Zi}$ and is mostly based on a careful analysis of all possible cases.

In Section 2 we collect all necessary preliminaries for 2-representation theory of the 2 -category $\mathscr{C}_{A}$. In Section 3 we prove some general results about 2 -representations of $\mathscr{C}_{A}$ under the additional assumption that the algebra $A$ has a non-zero projective injective module.

In Section 4, 5 and 6, we collect the proof of Theorem 1 in the case $A=\mathbb{k}(\bullet \rightarrow \bullet)$. In more details, Section 4 contains preliminaries on $\mathscr{C}_{A}$ for $A=\mathbb{k}(\bullet \rightarrow \bullet)$. Section 5 contains combinatorial results on certain integer matrices which allow us to specify three essentially different cases which we have to deal with. In Sections 6 we prove Theorem 1 for $A=\mathbb{k}(\bullet \rightarrow \bullet)$.

In Sections 7, 8, 9] and 10, we collect the proof of Theorem 11 in the second case

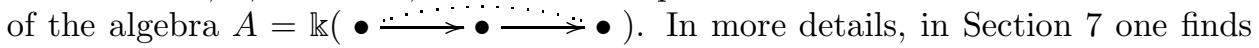
preliminaries on $\mathscr{C}_{A}$. Sections 8 and 9 are devoted to finding out which integer matrix captures the combinatorics of a faithful simple transitive 2-representation of $\mathscr{C}_{A}$. Finally, Sections 10 completes the proof of Theorem 1 for the algebra $\mathbb{k}(\bullet \stackrel{\cdots \cdots \cdots \cdots}{\longrightarrow} \bullet \stackrel{\cdots}{\longrightarrow})$.

Acknowledgment. The first author is partially supported by the Swedish Research Council. We thank Vanessa Miemietz for stimulating discussions.

\section{2-CATEGORY $\mathscr{C}_{A}$ AND ITS 2-REPRESENTATIONS}

2.1. Notation and conventions. Throughout the paper we work over an algebraically closed field $\mathbb{k}$ and abbreviate $\otimes_{\mathbb{k}}$ by $\otimes$. Unless explicitly stated otherwise, by a module, we mean a left module. We compose maps from right to left. For a 1-morphism $\mathrm{F}$, we denote by $\mathrm{id}_{\mathrm{F}}$ the identity 2-morphism for $\mathrm{F}$.

2.2. 2-category $\mathscr{C}_{A}$. We refer the reader to $\mathrm{Le}$, Mac, Maz for generalities on 2categories. A 2-category is a category which is enriched over the monoidal category Cat of small categories.

Let $A$ be a connected, basic, finite dimensional $\mathbb{k}$-algebra and $\mathcal{C}$ a small category equivalent to $A$-mod. Consider the 2 -category $\mathscr{C}_{A}$ (which depends on $\mathcal{C}$ ) defined as follows:

- $\mathscr{C}_{A}$ has one object $\mathrm{i}$ which we identify with $\mathcal{C}$;

- 1-morphisms in $\mathscr{C}_{A}$ are endofunctors of $\mathcal{C}$ isomorphic to functors given by tensoring with $A$ - $A$-bimodules from the additive closure of both ${ }_{A} A_{A}$ and ${ }_{A} A \otimes A_{A} ;$

- 2-morphisms in $\mathscr{C}_{A}$ are natural transformations of functors. 
The 2-category $\mathscr{C}_{A}$ is finitary in the sense of [MM1, Subsection 2.2].

2.3. 2-representations $\mathscr{C}_{A}$. We consider the 2-category $\mathscr{C}_{A}$-afmod of all finitary 2 -representations of $\mathscr{C}_{A}$. In this 2-category,

- objects are strict additive functorial actions of $\mathscr{C}_{A}$ on additive, idempotent split, Krull-Schmidt, $\mathbb{k}$-linear categories with finitely many isomorphism classes of indecomposable objects and finite dimensional morphism spaces;

- 1-morphisms are 2-natural transformations;

- 2-morphisms are modifications.

We refer the reader to MM3 for details. Two 2-representations are called equivalent provided that there is a 2-natural transformation between them whose restriction to each object is an equivalence of categories.

We also consider the 2-category $\mathscr{C}_{A}$-mod defined similarly using functorial action on categories equivalent to module categories of finite dimensional $\mathbb{k}$-algebras. There is the diagrammatically defined abelianization 2 -functor

$$
\div: \mathscr{C}_{A} \text {-afmod } \rightarrow \mathscr{C}_{A} \text {-mod, }
$$

see [MM2, Subsection 4.2] for details.

A finitary 2-representation of $\mathscr{C}_{A}$ is called transitive provided that, for any indecomposable objects $X$ and $Y$ in $\mathbf{M}(\mathrm{i})$, there is a 1-morphism $\mathrm{F}$ in $\mathscr{C}_{A}$ such that $Y$ is isomorphic to a direct summand of $\mathbf{M}(\mathrm{F}) X$.

A transitive 2-representation $\mathbf{M}$ is called simple provided that $\mathbf{M}(\mathbf{i})$ does not have non-zero proper $\mathscr{C}_{A}$-invariant ideals.

For simplicity, we will often use the module notation $\mathrm{F} X$ instead of the representation notation $\mathbf{M}(\mathrm{F}) X$.

2.4. Cells in $\mathscr{C}_{A}$. Let $1=e_{1}+e_{2}+\cdots+e_{n}$ be a primitive decomposition of $1 \in A$. Up to isomorphism, indecomposable 1-morphisms in $\mathscr{C}_{A}$ are given by tensoring with ${ }_{A} A_{A}$ or with ${ }_{A} A e_{i} \otimes e_{j} A_{A}$, where $i, j=1,2, \ldots, n$. We fix a representative $\mathrm{F}_{0}$ in the isomorphism class of 1-morphisms which correspond to tensoring with ${ }_{A} A_{A}$. For $i, j=1,2, \ldots, n$, we fix a representative $\mathrm{F}_{i j}$ in the isomorphism class of 1-morphisms which correspond to tensoring with ${ }_{A} A e_{i} \otimes e_{j} A_{A}$. The set of isomorphism classes of indecomposable 1-morphisms in $\mathscr{C}_{A}$ has the natural structure of a multisemigroup, see [MM2, Section 3] and KuMa. Combinatorics of this structure is encoded into so-called left, right and two-sided cells. For $\mathscr{C}_{A}$, the two sided-cells are

$$
\mathcal{J}_{0}:=\left\{\mathrm{F}_{0}\right\} \quad \text { and } \quad \mathcal{J}:=\left\{\mathrm{F}_{i j}: i, j=1,2, \ldots, n\right\} .
$$

The two-sided cell $\left\{\mathrm{F}_{0}\right\}$ is a left and a right cell as well. Other left cells are

$$
\left\{\mathrm{F}_{i j}: i=1,2, \ldots, n\right\}, \quad j=1,2, \ldots, n .
$$

Other right cells are

$$
\left\{\mathrm{F}_{i j}: j=1,2, \ldots, n\right\}, \quad i=1,2, \ldots, n .
$$

As usual, we have

$$
\mathrm{F}_{i j} \circ \mathrm{F}_{s t}=\mathrm{F}_{i t}^{\oplus \operatorname{dim}\left(e_{j} A e_{s}\right)} .
$$

We set

$$
\mathrm{F}:=\bigoplus_{i, j=1}^{n} \mathrm{~F}_{i j}
$$


and note that

$$
\mathrm{F} \circ \mathrm{F} \cong \mathrm{F}^{\oplus \operatorname{dim}(A)}
$$

All 1-morphisms in the additive closure of $\mathrm{F}$ are called projective endofunctors of C. Similarly for $A$-mod.

As usual, we will say that a pair $\left(\mathrm{F}_{i j}, \mathrm{~F}_{s t}\right)$ of 1-morphisms is a pair of adjoint 1-morphisms provided that there exist 2-morphisms

$$
\alpha: \mathrm{F}_{i j} \mathrm{~F}_{s t} \rightarrow \mathrm{F}_{0} \quad \text { and } \quad \beta: \mathrm{F}_{0} \rightarrow \mathrm{F}_{s t} \mathrm{~F}_{i j}
$$

such that

$$
\left(\alpha \circ_{0} \operatorname{id}_{\mathrm{F}_{i j}}\right) \circ_{1}\left(\operatorname{id}_{\mathrm{F}_{i j}} \circ_{0} \beta\right)=\operatorname{id}_{\mathrm{F}_{i j}} \quad \text { and } \quad\left(\operatorname{id}_{\mathrm{F}_{s t} \circ_{0}} \alpha\right) \circ_{1}\left(\beta \circ_{0} \operatorname{id}_{\mathrm{F}_{s t}}\right)=\operatorname{id}_{\mathrm{F}_{s t}} .
$$

The 2-category $\mathscr{C}_{A}$ is $\mathcal{J}$-simple in the sense that any non-zero two-sided 2 -ideal of $\mathscr{C}_{A}$ contains the identity 2-morphisms for all 1-morphisms given by projective endofunctors, see [MM2, Ag.

2.5. Cell 2-representations. The first example of a finitary 2-representation of $\mathscr{C}_{A}$ is the principal 2-representation $\mathbf{P}:=\mathscr{C}_{A}\left(\mathbf{i},{ }_{-}\right)$. This has a unique maximal $\mathscr{C}_{A}$-invariant ideal and the corresponding quotient is the cell 2-representation $\mathbf{C}_{\mathcal{L}}$, where $\mathcal{L}=\left\{\mathrm{F}_{0}\right\}$.

For any other left cell $\mathcal{L}$, the additive closure of elements in $\mathcal{L}$ gives a 2 -subrepresentation of $\mathbf{P}$. This 2 -subrepresentation again has a unique maximal $\mathscr{C}_{A}$-invariant ideal and the corresponding quotient is the cell 2-representation $\mathbf{C}_{\mathcal{L}}$. This latter cell 2 -representation is equivalent to the defining action of $\mathscr{C}_{A}$ on the category $A$-proj of projective objects in $A$-mod, see [MM1 for details.

2.6. Matrices in the Grothendieck group. Let $\mathbf{M}$ be a finitary 2-representation of $\mathscr{C}_{A}$ and $X_{1}, X_{2}, \ldots, X_{k}$ be a fixed complete and irredundant list of representatives of isomorphism classes of indecomposable objects in $\mathbf{M}(\mathbf{i})$. For a 1-morphism $\mathrm{G}$ in $\mathscr{C}_{A}$, we denote by [G] the $k \times k$ matrix with non-negative integer coefficients where, for $i, j=1,2, \ldots, k$, the coefficient in the intersection of the $i$-th row and the $j$-th column gives the number of indecomposable direct summands of $\mathbf{M}(\mathrm{G}) X_{j}$ which are isomorphic to $X_{i}$. Note that $[\mathrm{G} \oplus \mathrm{H}]=[\mathrm{G}]+[\mathrm{H}]$ and $[\mathrm{G} \circ \mathrm{H}]=[\mathrm{G}][\mathrm{H}]$.

2.7. Action on simple transitive 2-representations. The following statement is proved in [MM5, Lemma 12].

Lemma 2. Let $\mathbf{M}$ be a simple transitive 2-representation of $\mathscr{C}_{A}$. Then, for any non-zero object $X \in \overline{\mathbf{M}}(\mathrm{i})$, the object $\mathrm{F} X$ is projective in $\overline{\mathbf{M}}(\mathrm{i})$.

The following statement is proved in [MM5, Lemma 13].

Lemma 3. Let $B$ be a finite dimensional $\mathbb{k}$-algebra and $\mathrm{G}$ an exact endofunctor of $B$-mod. Assume that $\mathrm{G}$ sends each simple object of $B$-mod to a projective object. Then $\mathrm{G}$ is a projective functor. 


\section{Existence of A PROJECTIVE-INJECTIVE Module guARANTEes EXACTNESS OF THE ACTION}

3.1. Exactness of the action of some projective functors. Let $\mathbf{M}$ be a simple transitive 2-representation of $\mathscr{C}_{A}$. Consider its abelianization $\overline{\mathbf{M}}$. For $\overline{\mathbf{M}}(\mathrm{i})$, let $L_{1}, L_{2}, \ldots, L_{k}$ be a complete and irredundant list of representatives of isomorphism classes of simple objects. For $i \in\{1,2, \ldots, k\}$, denote by $P_{i}$ the indecomposable projective cover of $L_{i}$ and by $I_{i}$ the indecomposable injective envelope of $L_{i}$.

Lemma 4. Let $Q$ be a finite dimensional $\mathbb{k}$-algebra and $\mathrm{K}$ a right exact endofunctor of $Q$-mod. Then the following conditions are equivalent:

(a) The functor $\mathrm{K}$ sends projective objects to projective objects.

(b) The right adjoint $\mathrm{K}^{\prime}$ of $\mathrm{K}$ is exact.

Proof. By adjunction, for a projective generator $P \in Q$-mod, we have a natural isomorphism

$$
\operatorname{Hom}_{Q}\left(\mathrm{KP},_{-}\right) \cong \operatorname{Hom}_{Q}\left(P, \mathrm{~K}_{-}^{\prime}\right) .
$$

If $\mathrm{KP}$ is projective, the left hand side of (3) is exact. Hence the right hand side is also exact. As $P$ is a projective generator, the functor $\operatorname{Hom}_{Q}\left(P,_{-}\right)$detects any non-zero homology. This forces $\mathrm{K}^{\prime}$ to be exact. Therefore (a) implies (b).

Conversely, assume that $\mathrm{K}^{\prime}$ is exact. Then the right hand side of (3) is exact. Hence the left hand side is exact. This means that $\mathrm{KP}$ is projective. Therefore (b) implies (a). The claim follows.

Lemma 5. Assume that there exist $s, t \in\{1,2, \ldots, n\}$ such that the left $A$-modules $A e_{s}$ and $\operatorname{Hom}_{\mathbb{k}}\left(e_{t} A, \mathbb{k}\right)$ are isomorphic. Then, for any $i \in\{1,2, \ldots, n\}$, the pair $\left(\mathrm{F}_{i t}, \mathrm{~F}_{\text {si }}\right)$ is a pair of adjoint 1-morphisms.

Proof. The functor $\mathrm{F}_{i t}$ is given by tensoring with the $A$ - $A$-bimodule $A e_{i} \otimes e_{t} A$. The right adjoint of this functor is thus the functor $\operatorname{Hom}_{A}\left(A e_{i} \otimes e_{t} A,_{-}\right)$. By the computation in [MM1, Subsection 7.3], the exact functor $\operatorname{Hom}_{A}\left(A e_{i} \otimes e_{t} A,_{-}\right)$is isomorphic to the functor of tensoring with the $A$ - $A$-bimodule

$$
\operatorname{Hom}_{\mathbb{k}}\left(e_{t} A, \mathbb{k}\right) \otimes e_{i} A .
$$

The injective $A$-module $I_{t} \cong \operatorname{Hom}_{\mathbb{k}}\left(e_{t} A, \mathbb{k}\right)$ is isomorphic to the projective $A$-module $A e_{s}$, by assumption. Therefore $\operatorname{Hom}_{\mathbb{k}}\left(e_{t} A, \mathbb{k}\right) \otimes e_{i} A$ is isomorphic to $A e_{s} \otimes e_{i} A$. This means that $\mathrm{F}_{s i}$ is isomorphic to the right adjoint of $\mathrm{F}_{i t}$. The claim follows.

Corollary 6. Assume that there exist $s, t \in\{1,2, \ldots, n\}$ such that the left $A$ modules $A e_{s}$ and $\operatorname{Hom}_{\mathbb{k}}\left(e_{t} A, \mathbb{k}\right)$ are isomorphic. Then, for any $i \in\{1,2, \ldots, n\}$ and any 2-representation $\mathbf{N}$ of $\mathscr{C}_{A}$, the pair $\left(\mathbf{N}\left(\mathrm{F}_{i t}\right), \mathbf{N}\left(\mathrm{F}_{s i}\right)\right)$ is a pair of adjoint functors.

Proof. This follows directly from Lemma 5 and definitions.

Corollary 7. Assume that there exist $s, t \in\{1,2, \ldots, n\}$ such that the left $A$-modules $A e_{s}$ and $\operatorname{Hom}_{\mathbb{k}}\left(e_{t} A, \mathbb{k}\right)$ are isomorphic. Then, for any $i \in\{1,2, \ldots, n\}$ and any finitary 2-representation $\mathbf{N}$ of $\mathscr{C}_{A}$, the functor $\overline{\mathbf{N}}\left(\mathrm{F}_{\text {si }}\right)$ is exact.

Proof. This follows from the definitions by combining Lemma 4 and Corollary 6 . 


\subsection{Auxiliary lemma.}

Lemma 8. Let $Q$ be a finite dimensional $\mathbb{k}$-algebra and $\mathrm{K}, \mathrm{H}$ and $\mathrm{G}$ be two endofunctor of $Q$-mod. Assume that:

(a) $\mathrm{H}$ is a projective functor;

(b) $\mathrm{K}$ is right exact;

(c) $\mathrm{K}$ sends projective objects to projective objects;

(d) $\mathrm{K} \circ \mathrm{H} \cong \mathrm{G}$.

Then $\mathrm{G}$ is a projective functor.

Proof. By assumption (国), the functor $\mathrm{H}$ is given by tensoring with the $Q-Q$ bimodule $X \otimes Y$, for some projective left $Q$-module $X$ and some projective right $Q$-module $Y$. By assumption (b), $\mathrm{K}$ is given by tensoring with some $Q$ - $Q$-bimodule $V$. Using assumption (d), the $Q$ - $Q$-bimodule that determines the functor $\mathrm{G}$ is given by

$$
V \otimes_{Q}(X \otimes Y) \cong\left(V \otimes_{Q} X\right) \otimes Y .
$$

By assumption (IC), $V \otimes_{Q} X$ is a projective left $Q$-module. This implies that (4) is a projective $Q$ - $Q$-bimodule and hence $\mathrm{G}$ is a projective functor.

\subsection{Exactness of the action.}

Proposition 9. Assume that there exist $s, t \in\{1,2, \ldots, n\}$ such that the left $A$ modules $A e_{s}$ and $\operatorname{Hom}_{\mathbb{k}}\left(e_{t} A, \mathbb{k}\right)$ are isomorphic. Let $\mathbf{M}$ be a simple transitive 2representation of $\mathscr{C}_{A}$. Then the functor $\overline{\mathbf{M}}(\mathrm{F})$ is exact.

Proof. Let $B$ be a finite dimensional algebra such that $\overline{\mathbf{M}}(i)$ is equivalent to $B$-mod.

For $i \in\{1,2, \ldots, n\}$, consider the 1-morphism $\mathrm{F}_{s i}$. By Corollary 7 , the functor $\overline{\mathbf{M}}\left(\mathrm{F}_{s i}\right)$ is exact. By Lemma 2, $\overline{\mathbf{M}}\left(\mathrm{F}_{s i}\right)$ sends any object in $\overline{\mathbf{M}}(\mathrm{i})$ to a projective object. Therefore, by Lemma 3, $\overline{\mathbf{M}}\left(\mathrm{F}_{s i}\right)$ is a projective endofunctor of $B$-mod.

Now, for any $j \in\{1,2, \ldots, n\}$, we have

$$
\mathrm{F}_{j s} \circ \mathrm{F}_{s i} \cong \mathrm{F}_{j i}^{\oplus k}
$$

where $k=\operatorname{dim}\left(e_{s} A e_{s}\right)>0$. Therefore $\overline{\mathbf{M}}\left(\mathrm{F}_{j i}^{\oplus k}\right)$ is a projective functor for $B$-mod by Lemma 8 , By additivity, $\overline{\mathbf{M}}\left(\mathrm{F}_{j i}\right)$ is a projective functor for $B$-mod as well. In particular, $\overline{\mathbf{M}}\left(\mathrm{F}_{j i}\right)$ is exact. The claim follows.

\section{The AlgEBRA $\mathbb{k}(\bullet \rightarrow \bullet)$}

Let $\mathbb{k}$ be an algebraically closed field. Denote by $A$ the path algebra, over $\mathbb{k}$, of the quiver $1 \stackrel{\alpha}{\longrightarrow} 2$. The algebra $A$ has basis $e_{1}, e_{2}$ and $\alpha$ and the multiplication table $(x, y) \mapsto x \cdot y$ is given by:

\begin{tabular}{c||c|c|c}
$x \backslash y$ & $e_{1}$ & $e_{2}$ & $\alpha$ \\
\hline \hline$e_{1}$ & $e_{1}$ & 0 & 0 \\
\hline$e_{2}$ & 0 & $e_{2}$ & $\alpha$ \\
\hline$\alpha$ & $\alpha$ & 0 & 0
\end{tabular}

Note that $e_{1} A e_{2}=0$ as $A$ contains no paths from 2 to 1 . Note also that the left $A$-modules $A e_{1}$ and $\operatorname{Hom}_{\mathbb{k}}\left(e_{2} A, \mathbb{k}\right)$ are isomorphic. 
Let $\mathcal{C}$ be a small category equivalent to $A$-mod. Consider the corresponding finitary 2-category $\mathscr{C}_{A}$. Up to isomorphism, indecomposable 1-morphisms in $\mathscr{C}_{A}$ are $\mathrm{F}_{0}$ and $\mathrm{F}_{i j}$, where $i, j=1,2$. Note that formula (2) for $A$ reads $\mathrm{F} \circ \mathrm{F}=\mathrm{F}^{\oplus 3}$. Using (11), the table of compositions for the functors $\mathrm{F}_{i j}$ (up to isomorphism) is as follows:

\begin{tabular}{c||c|c|c|c}
$\circ$ & $\mathrm{F}_{11}$ & $\mathrm{~F}_{12}$ & $\mathrm{~F}_{21}$ & $\mathrm{~F}_{22}$ \\
\hline $\mathrm{F}_{11}$ & $\mathrm{~F}_{11}$ & $\mathrm{~F}_{12}$ & 0 & 0 \\
\hline $\mathrm{F}_{12}$ & $\mathrm{~F}_{11}$ & $\mathrm{~F}_{12}$ & $\mathrm{~F}_{11}$ & $\mathrm{~F}_{12}$ \\
\hline $\mathrm{F}_{21}$ & $\mathrm{~F}_{21}$ & $\mathrm{~F}_{22}$ & 0 & 0 \\
\hline $\mathrm{F}_{22}$ & $\mathrm{~F}_{21}$ & $\mathrm{~F}_{22}$ & $\mathrm{~F}_{21}$ & $\mathrm{~F}_{22}$
\end{tabular}

Set $\mathcal{J}_{0}:=\left\{\mathrm{F}_{0}\right\}$ and $\mathcal{J}:=\left\{\mathrm{F}_{i j}: i, j=1,2\right\}$. Note that the 2-category $\mathscr{C}_{A}$ is not weakly fiat in the sense of [MM2, [MM6] as the algebra $A$ is not self-injective.

As $\mathscr{C}_{A}$ is $\mathcal{J}$-simple and $A$ has trivial center, the only proper non-zero quotient of $\mathscr{C}_{A}$ contains just the identity 1-morphism (up to isomorphism) and its scalar endomorphisms (cf. [MM3]). Therefore this quotient is fiat with strongly regular $\mathcal{J}$-classes and hence it has a unique, up to equivalence, simple transitive 2-representation, namely $\mathbf{C}_{\mathcal{L}_{0}}$, where $\mathcal{L}_{0}=\mathcal{J}_{0}$, see [MM5, Theorem 18]. This means that, in order to prove Theorem 1 for $A$, it is enough to consider faithful 2-representations of $\mathscr{C}_{A}$.

From the formula

$$
\operatorname{Hom}_{A-A}\left(A e_{i} \otimes e_{j} A, A e_{s} \otimes e_{t} A\right) \cong e_{i} A e_{s} \otimes e_{t} A e_{j},
$$

for all $i, j, s, t \in\{1,2\}$, we get the following table of $\operatorname{Hom}_{\mathscr{C}_{A}(\mathrm{i})}(X, Y)$ (up to isomorphism), where $X$ and $Y$ are indecomposable 1-morphisms as specified in the table:

\begin{tabular}{c||c|c|c|c}
$X \backslash Y$ & $\mathrm{~F}_{11}$ & $\mathrm{~F}_{12}$ & $\mathrm{~F}_{21}$ & $\mathrm{~F}_{22}$ \\
\hline \hline $\mathrm{F}_{11}$ & $\mathbb{k}$ & $\mathbb{k}$ & 0 & 0 \\
\hline $\mathrm{F}_{12}$ & 0 & $\mathbb{k}$ & 0 & 0 \\
\hline $\mathrm{F}_{21}$ & $\mathbb{k}$ & $\mathbb{k}$ & $\mathbb{k}$ & $\mathbb{k}$ \\
\hline $\mathrm{F}_{22}$ & 0 & $\mathbb{k}$ & 0 & $\mathbb{k}$
\end{tabular}

\section{INTEGER MATRICES FOR $\mathbb{k}(\bullet \rightarrow \bullet)$}

5.1. Integer matrices satisfying $M^{2}=3 M$. In this section we classify all square matrices $M$ with positive integer coefficients which satisfy $M^{2}=3 M$.

Proposition 10. Let $M$ be $a k \times k$ matrix, for some $k$, with positive integer coefficients, satisfying $M^{2}=3 M$. Then $M$ is one of the following matrices:

$$
\begin{gathered}
M_{1}:=(3), \quad M_{2}:=\left(\begin{array}{ll}
2 & 1 \\
2 & 1
\end{array}\right), \quad M_{3}:=\left(\begin{array}{ll}
2 & 2 \\
1 & 1
\end{array}\right), \quad M_{4}:=\left(\begin{array}{ll}
1 & 1 \\
2 & 2
\end{array}\right), \\
M_{5}:=\left(\begin{array}{ll}
1 & 2 \\
1 & 2
\end{array}\right), \quad M_{6}:=\left(\begin{array}{lll}
1 & 1 & 1 \\
1 & 1 & 1 \\
1 & 1 & 1
\end{array}\right) .
\end{gathered}
$$

Proof. Clearly, we have $M_{i}^{2}=3 M_{i}$, for each $i=1,2,3,4,5,6$. So, we need to show that no other square matrix with positive integer coefficients satisfies $M^{2}=3 M$.

Let $M$ be a $k \times k$ matrix, for some $k$, with positive integer coefficients satisfying $M^{2}=3 M$. Then $M$ is diagonalizable (as $x^{2}-3 x$ has no multiple roots) and the only possible eigenvalues for $M$ are 0 and 3 . From the Perron-Frobenius theorem it 
follows that the Perron-Frobenius eigenvalue 3 must have multiplicity one. Therefore $M$ has rank one and trace three. As all entries in $M$ are positive integers, we get $k \leq 3$.

If $k=1$, then, clearly, $M=M_{1}$.

If $k=3$, then all diagonal entries in $M$ are 1 . As all $2 \times 2$ minors in $M$ should have determinant zero and positive integer entries, it follows that all entries in $M$ are 1 and thus $M=M_{6}$.

If $k=2$, then the two diagonal entries in $M$ are 1 and 2. As the determinant of $M$ is zero, the two remaining entries are also 1 and 2 . Therefore $M=M_{i}$, for some $i \in\{2,3,4,5\}$.

5.2. The matrix $[\mathrm{F}]$ for a faithful simple transitive 2-representation. Let $\mathbf{M}$ be a finitary, simple, transitive and faithful 2-representation of $\mathscr{C}_{A}$. Let $M:=[\mathrm{F}]$ be the matrix of $\mathbf{M}(\mathrm{F})$ and, for $i, j=1,2$, let $M_{i j}:=\left[\mathrm{F}_{i j}\right]$ be the matrix of $\mathbf{M}\left(\mathrm{F}_{i j}\right)$. Note that $M=M_{11}+M_{12}+M_{21}+M_{22}$.

The symmetric group $S_{k}$ acts on $\operatorname{Mat}_{k \times k}(\mathbb{Z})$ by conjugation with permutation matrices. This action corresponds to permutation of basis elements, whenever the matrix on which we act represents an endomorphism of some free $\mathbb{Z}$-module. We will call this action the permutation action.

Proposition 11. In order to respect the multiplication rule (5), up to the permutation action, we have the following three possibilities:

(a) $M=M_{2}$ and

$$
M_{11}=\left(\begin{array}{ll}
1 & 0 \\
0 & 0
\end{array}\right), M_{12}=\left(\begin{array}{ll}
1 & 1 \\
0 & 0
\end{array}\right), M_{21}=\left(\begin{array}{ll}
0 & 0 \\
1 & 0
\end{array}\right), M_{22}=\left(\begin{array}{ll}
0 & 0 \\
1 & 1
\end{array}\right) .
$$

(b) $M=M_{3}$ and

$$
M_{11}=\left(\begin{array}{ll}
0 & 1 \\
0 & 1
\end{array}\right), M_{12}=\left(\begin{array}{ll}
1 & 0 \\
1 & 0
\end{array}\right), M_{21}=\left(\begin{array}{ll}
0 & 1 \\
0 & 0
\end{array}\right), M_{22}=\left(\begin{array}{ll}
1 & 0 \\
0 & 0
\end{array}\right) .
$$

(c) $M=M_{6}$ and

$$
\begin{aligned}
& M_{11}=\left(\begin{array}{lll}
1 & 0 & 0 \\
1 & 0 & 0 \\
0 & 0 & 0
\end{array}\right), M_{12}=\left(\begin{array}{lll}
0 & 1 & 1 \\
0 & 1 & 1 \\
0 & 0 & 0
\end{array}\right), \\
& M_{21}=\left(\begin{array}{lll}
0 & 0 & 0 \\
0 & 0 & 0 \\
1 & 0 & 0
\end{array}\right), M_{22}=\left(\begin{array}{lll}
0 & 0 & 0 \\
0 & 0 & 0 \\
0 & 1 & 1
\end{array}\right) .
\end{aligned}
$$

Proof. As $\mathbf{M}$ is simple, transitive and faithful, we get that $M$ has positive integer entries. As $\mathrm{F} \circ \mathrm{F}=\mathrm{F}^{\oplus 3}$, we have $M=M_{i}$ for some $i \in\{1,2,3,4,5,6\}$, by Proposition 10. As $M$ is the sum of four non-zero matrices (corresponding to all $\mathrm{F}_{i j}$ ) each of which has non-negative integer entries, we have $M \neq M_{1}$. The case $M=M_{4}$ reduces to the case $M=M_{3}$ by swapping the basis elements. The case $M=M_{5}$ reduces to the case $M=M_{2}$ by swapping the basis elements. It is easy

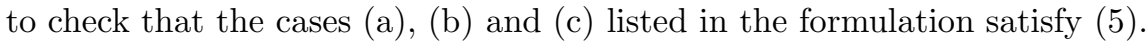

Assume $M=M_{2}$. Note, from (5), that $\mathrm{F}_{11}, \mathrm{~F}_{12}$ and $\mathrm{F}_{22}$ are idempotent, while $\mathrm{F}_{21}$ is nilpotent. Therefore $M_{11}, M_{12}, M_{22}$ must have non-zero diagonals, while the diagonal for $M_{21}$ should be zero. From $M_{11} M_{22}=0$ it follows that $M_{11}$ and $M_{22}$ 
cannot have common diagonal entries. In any case, this means that $M_{12}$ has the non-zero diagonal entry in the left upper corner. Let us first assume the following:

$$
M_{11}=\left(\begin{array}{ll}
0 & * \\
* & 1
\end{array}\right), M_{12}=\left(\begin{array}{cc}
1 & * \\
* & 0
\end{array}\right), M_{21}=\left(\begin{array}{cc}
0 & * \\
* & 0
\end{array}\right), M_{22}=\left(\begin{array}{cc}
1 & * \\
* & 0
\end{array}\right) .
$$

From $M_{11} M_{21}=0$, we get:

$$
M_{11}=\left(\begin{array}{ll}
0 & 0 \\
0 & 1
\end{array}\right), M_{12}=\left(\begin{array}{ll}
1 & 0 \\
* & 0
\end{array}\right), M_{21}=\left(\begin{array}{ll}
0 & 1 \\
0 & 0
\end{array}\right), M_{22}=\left(\begin{array}{ll}
1 & 0 \\
* & 0
\end{array}\right) .
$$

This, however, contradicts $M_{11} M_{12}=M_{12}$. Now assume

$$
M_{11}=\left(\begin{array}{ll}
1 & * \\
* & 0
\end{array}\right), M_{12}=\left(\begin{array}{cc}
1 & * \\
* & 0
\end{array}\right), M_{21}=\left(\begin{array}{cc}
0 & * \\
* & 0
\end{array}\right), M_{22}=\left(\begin{array}{cc}
0 & * \\
* & 1
\end{array}\right) .
$$

From $M_{11} M_{21}=M_{11} M_{22}=0$, we get:

$$
M_{11}=\left(\begin{array}{ll}
1 & 0 \\
* & 0
\end{array}\right), M_{12}=\left(\begin{array}{ll}
1 & 1 \\
* & 0
\end{array}\right), M_{21}=\left(\begin{array}{ll}
0 & 0 \\
* & 0
\end{array}\right), M_{22}=\left(\begin{array}{cc}
0 & 0 \\
* & 1
\end{array}\right) .
$$

From $M_{12} M_{22}=M_{12}$, we get:

$$
M_{11}=\left(\begin{array}{ll}
1 & 0 \\
0 & 0
\end{array}\right), M_{12}=\left(\begin{array}{ll}
1 & 1 \\
0 & 0
\end{array}\right), M_{21}=\left(\begin{array}{ll}
0 & 0 \\
1 & 0
\end{array}\right), M_{22}=\left(\begin{array}{ll}
0 & 0 \\
1 & 1
\end{array}\right) .
$$

Assume $M=M_{3}$. Note, from (5), that $\mathrm{F}_{11}, \mathrm{~F}_{12}$ and $\mathrm{F}_{22}$ are idempotent, while $\mathrm{F}_{21}$ is nilpotent. Therefore $M_{11}, M_{12}, M_{22}$ must have non-zero diagonals, while the diagonal for $M_{21}$ should be zero. From $M_{11} M_{22}=0$ it follows that $M_{11}$ and $M_{22}$ cannot have common diagonal entries. In any case this means that $M_{12}$ has the non-zero diagonal entry in the left upper corner. Let us first assume the following:

$$
M_{11}=\left(\begin{array}{ll}
1 & * \\
* & 0
\end{array}\right), M_{12}=\left(\begin{array}{cc}
1 & * \\
* & 0
\end{array}\right), M_{21}=\left(\begin{array}{cc}
0 & * \\
* & 0
\end{array}\right), M_{22}=\left(\begin{array}{ll}
0 & * \\
* & 1
\end{array}\right) .
$$

From $M_{11} M_{21}=0$, we get:

$$
M_{11}=\left(\begin{array}{ll}
1 & 0 \\
0 & 0
\end{array}\right), M_{12}=\left(\begin{array}{ll}
1 & * \\
0 & 0
\end{array}\right), M_{21}=\left(\begin{array}{ll}
0 & 0 \\
1 & 0
\end{array}\right), M_{22}=\left(\begin{array}{ll}
0 & * \\
0 & 1
\end{array}\right) .
$$

This, however, contradicts $M_{21} M_{12}=M_{22}$. Now assume the following:

$$
M_{11}=\left(\begin{array}{ll}
0 & * \\
* & 1
\end{array}\right), M_{12}=\left(\begin{array}{cc}
1 & * \\
* & 0
\end{array}\right), M_{21}=\left(\begin{array}{cc}
0 & * \\
* & 0
\end{array}\right), M_{22}=\left(\begin{array}{cc}
1 & * \\
* & 0
\end{array}\right) .
$$

From $M_{11} M_{21}=M_{11} M_{22}=0$, we get:

$$
M_{11}=\left(\begin{array}{ll}
0 & * \\
0 & 1
\end{array}\right), M_{12}=\left(\begin{array}{ll}
1 & * \\
1 & 0
\end{array}\right), M_{21}=\left(\begin{array}{cc}
0 & * \\
0 & 0
\end{array}\right), M_{22}=\left(\begin{array}{ll}
1 & * \\
0 & 0
\end{array}\right) .
$$

From $M_{11} M_{12}=M_{12}$, we get:

$$
M_{11}=\left(\begin{array}{ll}
0 & 1 \\
0 & 1
\end{array}\right), M_{12}=\left(\begin{array}{ll}
1 & 0 \\
1 & 0
\end{array}\right), M_{21}=\left(\begin{array}{ll}
0 & 1 \\
0 & 0
\end{array}\right), M_{22}=\left(\begin{array}{ll}
1 & 0 \\
0 & 0
\end{array}\right) .
$$

Assume $M=M_{6}$. Note, from (5), that $\mathrm{F}_{11}, \mathrm{~F}_{12}$ and $\mathrm{F}_{22}$ are idempotent, while $\mathrm{F}_{21}$ is nilpotent. Therefore $M_{11}, M_{12}, M_{22}$ must have non-zero diagonals, while the diagonal for $M_{21}$ should be zero. Therefore, up to permutation of basis vectors, we 
may assume that

$$
\begin{aligned}
& M_{11}=\left(\begin{array}{lll}
1 & * & * \\
* & 0 & * \\
* & * & 0
\end{array}\right), M_{12}=\left(\begin{array}{lll}
0 & * & * \\
* & 1 & * \\
* & * & 0
\end{array}\right), \\
& M_{21}=\left(\begin{array}{lll}
0 & * & * \\
* & 0 & * \\
* & * & 0
\end{array}\right), M_{22}=\left(\begin{array}{lll}
0 & * & * \\
* & 0 & * \\
* & * & 1
\end{array}\right) .
\end{aligned}
$$

From $M_{11} M_{21}=M_{11} M_{22}=0$ we thus get that the last column of $M_{11}$ must be zero and the first row of both $M_{21}$ and $M_{22}$ must be zero. Since the $M_{i j}$ 's add up to $M$, the rightmost element in the first row of $M_{12}$ must be 1:

$$
\begin{aligned}
& M_{11}=\left(\begin{array}{lll}
1 & * & 0 \\
* & 0 & 0 \\
* & * & 0
\end{array}\right), M_{12}=\left(\begin{array}{lll}
0 & * & 1 \\
* & 1 & * \\
* & * & 0
\end{array}\right), \\
& M_{21}=\left(\begin{array}{lll}
0 & 0 & 0 \\
* & 0 & * \\
* & * & 0
\end{array}\right), M_{22}=\left(\begin{array}{lll}
0 & 0 & 0 \\
* & 0 & * \\
* & * & 1
\end{array}\right) .
\end{aligned}
$$

From $M_{11} M_{12}=M_{12}$ it follows that the second row of $M_{11}$ cannot be zero, which yields:

$$
\begin{aligned}
& M_{11}=\left(\begin{array}{lll}
1 & * & 0 \\
1 & 0 & 0 \\
* & * & 0
\end{array}\right), M_{12}=\left(\begin{array}{lll}
0 & * & 1 \\
0 & 1 & * \\
* & * & 0
\end{array}\right), \\
& M_{21}=\left(\begin{array}{lll}
0 & 0 & 0 \\
0 & 0 & * \\
* & * & 0
\end{array}\right), M_{22}=\left(\begin{array}{lll}
0 & 0 & 0 \\
0 & 0 & * \\
* & * & 1
\end{array}\right) .
\end{aligned}
$$

Now $M_{11} M_{12}=M_{12}$ implies that the first and the second rows of $M_{12}$ should coincide, moreover, the first element in the third row in $M_{12}$ should be zero and also the third row in $M_{11}$ and thus also in $M_{12}$ must be zero:

$$
\begin{aligned}
& M_{11}=\left(\begin{array}{lll}
1 & 0 & 0 \\
1 & 0 & 0 \\
0 & 0 & 0
\end{array}\right), M_{12}=\left(\begin{array}{lll}
0 & 1 & 1 \\
0 & 1 & 1 \\
0 & 0 & 0
\end{array}\right), \\
& M_{21}=\left(\begin{array}{lll}
0 & 0 & 0 \\
0 & 0 & 0 \\
* & * & 0
\end{array}\right), M_{22}=\left(\begin{array}{lll}
0 & 0 & 0 \\
0 & 0 & 0 \\
* & * & 1
\end{array}\right) .
\end{aligned}
$$

Now, $M_{21} M_{11}=M_{21}$ gives:

$$
\begin{aligned}
& M_{11}=\left(\begin{array}{lll}
1 & 0 & 0 \\
1 & 0 & 0 \\
0 & 0 & 0
\end{array}\right), M_{12}=\left(\begin{array}{lll}
0 & 1 & 1 \\
0 & 1 & 1 \\
0 & 0 & 0
\end{array}\right), \\
& M_{21}=\left(\begin{array}{lll}
0 & 0 & 0 \\
0 & 0 & 0 \\
* & 0 & 0
\end{array}\right), M_{22}=\left(\begin{array}{lll}
0 & 0 & 0 \\
0 & 0 & 0 \\
* & 1 & 1
\end{array}\right) .
\end{aligned}
$$

Finally, $M_{12} M_{21}=M_{11}$ gives:

$$
\begin{aligned}
& M_{11}=\left(\begin{array}{lll}
1 & 0 & 0 \\
1 & 0 & 0 \\
0 & 0 & 0
\end{array}\right), M_{12}=\left(\begin{array}{lll}
0 & 1 & 1 \\
0 & 1 & 1 \\
0 & 0 & 0
\end{array}\right), \\
& M_{21}=\left(\begin{array}{lll}
0 & 0 & 0 \\
0 & 0 & 0 \\
1 & 0 & 0
\end{array}\right), M_{22}=\left(\begin{array}{lll}
0 & 0 & 0 \\
0 & 0 & 0 \\
0 & 1 & 1
\end{array}\right) .
\end{aligned}
$$




\section{Proof of Theorem 1 For $\mathbb{k}(\bullet \rightarrow \bullet)$}

Let $\mathbf{M}$ be a simple transitive 2-representation of $\mathscr{C}_{A}$. Let $B$ be a basic finite dimensional algebra such that $\mathbf{M}(\mathbf{i})$ is equivalent to $B$-proj.

As the left $A$-modules $A e_{1}$ and $\operatorname{Hom}_{\mathbb{k}}\left(e_{2} A, \mathbb{k}\right)$ are isomorphic, from Proposition 9 it follows that the functor $\overline{\mathbf{M}}(\mathrm{F})$ is exact. From Lemmata 2 and 3 we thus obtain that $\overline{\mathbf{M}}(\mathrm{F})$ is a projective endofunctor of $B$-mod.

Case 1. Assume that $M=M_{3}$ and the $M_{i j}$ 's are thus given by Proposition 11(b). Let $\overline{\mathbf{M}}$ be the abelianization of $\mathbf{M}$. As usual, we write $P_{1}$ and $P_{2}$ for indecomposable projectives in $\mathbf{M}(\mathbf{i})$ and $L_{1}$ and $L_{2}$ for their respective simple tops. Let $\epsilon_{1}$ and $\epsilon_{2}$ be the corresponding primitive idempotents in $B$. For $i, j=1,2$, denote by $\mathrm{G}_{i j}$ an endofunctor of $\overline{\mathbf{M}}(\mathrm{i})$ which corresponds to tensoring with $B \epsilon_{i} \otimes \epsilon_{j} B$.

From the form of $M_{21}$, we see that $\mathrm{F}_{21}$ acts via $\mathrm{G}_{12}$. Similarly, $\mathrm{F}_{22}$ acts via $\mathrm{G}_{11}$. From the matrices $M_{21}$ and $M_{22}$ it follows that

$$
\left[P_{1}: L_{1}\right]=1, \quad\left[P_{1}: L_{2}\right]=0, \quad\left[P_{2}: L_{1}\right]=0, \quad\left[P_{2}: L_{2}\right]=1 .
$$

This means that $B \cong \mathbb{k} \oplus \mathbb{k}$. Therefore all $\mathrm{G}_{i j}$ are isomorphisms between the corresponding $\mathbb{k}$-mod components. From the matrices $M_{12}$ and $M_{21}$ it thus follows directly that there are no nonzero homomorphisms from $F_{21}$ to $F_{12}$. This contradicts (7) and hence Case 1 cannot occur.

Case 2. Assume that $M=M_{2}$ and the $M_{i j}$ 's are thus given by Proposition 11(a). Let $\overline{\mathbf{M}}$ be the abelianization of $\mathbf{M}$. As usual, we write $P_{1}$ and $P_{2}$ for indecomposable projectives in $\mathbf{M}(i)$ and $L_{1}$ and $L_{2}$ for their respective simple tops. Let $\epsilon_{1}$ and $\epsilon_{2}$ be the corresponding primitive idempotents in $B$. For $i, j=1,2$, denote by $\mathrm{G}_{i j}$ an endofunctor of $\overline{\mathbf{M}}(\mathrm{i})$ which corresponds to tensoring with $B \epsilon_{i} \otimes \epsilon_{j} B$.

From the form of $M_{11}$, we see that $\mathrm{F}_{11}$ acts via $\mathrm{G}_{11}$. Similarly, $\mathrm{F}_{21}$ acts via $\mathrm{G}_{21}$.

From the form of $M_{12}$, we see that $\mathrm{F}_{12}$ acts either via $\mathrm{G}_{12}$ or via $\mathrm{G}_{11}$ or via $\mathrm{G}_{12} \oplus \mathrm{G}_{11}$. However, we already know that the matrix of $\mathrm{G}_{11}$ is $M_{11}$. This leaves us with possibilities $\mathrm{G}_{12}$ or $\mathrm{G}_{12} \oplus \mathrm{G}_{11}$ for $\mathrm{F}_{12}$.

Assume that $\mathrm{F}_{12}$ acts via $\mathrm{G}_{12} \oplus \mathrm{G}_{11}$. We already know the matrix of $\mathrm{G}_{11}$, so the matrix of $\mathrm{G}_{12}$ is

This and the matrix $M_{11}$ imply that

$$
\left(\begin{array}{ll}
0 & 1 \\
0 & 0
\end{array}\right)
$$

$$
\mathrm{G}_{11} P_{1} \cong P_{1}, \quad \mathrm{G}_{11} P_{2}=0, \quad \mathrm{G}_{12} P_{1}=0, \quad \mathrm{G}_{12} P_{2} \cong P_{2} .
$$

Therefore

$$
\left[P_{1}: L_{1}\right]=1, \quad\left[P_{1}: L_{2}\right]=0, \quad\left[P_{2}: L_{1}\right]=0, \quad\left[P_{2}: L_{2}\right]=1
$$

and we have $B \cong \mathbb{k} \oplus \mathbb{k}$. This leads to the same contradiction as in Case 1 above. Therefore $F_{12}$ acts via $G_{12}$. Similarly one shows that $F_{22}$ acts via $G_{22}$.

From the matrices for all $\mathrm{G}_{i j}$ 's it follows that the Cartan matrices of $A$ and $B$ coincide which implies that $A$ and $B$ are isomorphic (that is special for our case, but the algebra $A$ is very small, so this claim is clear). Furthermore, all $\mathrm{F}_{i j}$ 's act via the corresponding $\mathrm{G}_{i j}$. It now follows by the usual arguments, see MM5, Proposition 9], that $\overline{\mathbf{M}}$ is equivalent to a cell 2-representation of $\mathscr{C}_{A}$. 
Case 3. Assume that $M=M_{6}$ and the $M_{i j}$ 's are thus given by Proposition 11( (C). Let $\overline{\mathbf{M}}$ be the abelianization of $\mathbf{M}$. As usual, we write $P_{1}, P_{2}$ and $P_{3}$ for indecomposable projectives in $\mathbf{M}(i)$ and $L_{1}, L_{2}$ and $L_{3}$ for their respective simple tops. Let $\epsilon_{1}, \epsilon_{2}$ and $\epsilon_{3}$ be the corresponding primitive idempotents in $B$. For $i, j=1,2,3$, denote by $\mathbf{G}_{i j}$ an endofunctor of $\overline{\mathbf{M}}(\mathbf{i})$ which corresponds to tensoring with $B \epsilon_{i} \otimes \epsilon_{j} B$.

From the form of $M_{21}$, we see that $\mathrm{F}_{21}$ acts via $\mathrm{G}_{31}$. From the form of $M_{11}$, we see that $\mathrm{F}_{11}$ acts via $\mathrm{G}_{11} \oplus \mathrm{G}_{21}$. This implies

$$
\left[P_{1}: L_{1}\right]=1, \quad\left[P_{2}: L_{1}\right]=\left[P_{3}: L_{1}\right]=0 .
$$

From the form of $M_{22}$, we see that $\mathrm{F}_{22}$ acts either via $\mathrm{G}_{32}$ or via $\mathrm{G}_{33}$ or via $\mathrm{G}_{32} \oplus \mathrm{G}_{33}$. In the latter case, we have that the matrices of $\mathrm{G}_{32}$ and $\mathrm{G}_{33}$ are, respectively:

$$
\left(\begin{array}{ccc}
0 & 0 & 0 \\
0 & 0 & 0 \\
0 & 1 & 0
\end{array}\right) \text { and }\left(\begin{array}{ccc}
0 & 0 & 0 \\
0 & 0 & 0 \\
0 & 0 & 1
\end{array}\right) \text {. }
$$

It follows that

$$
\left[P_{2}: L_{2}\right]=1, \quad\left[P_{1}: L_{2}\right]=\left[P_{3}: L_{2}\right]=0
$$

and

$$
\left[P_{3}: L_{3}\right]=1, \quad\left[P_{1}: L_{3}\right]=\left[P_{2}: L_{3}\right]=0 .
$$

This implies that $B \cong \mathbb{k} \oplus \mathbb{k} \oplus \mathbb{k}$ and leads to a similar contradiction as in Case 1 .

Subcase 3.1. Assume that $\mathrm{F}_{22}$ acts via $\mathrm{G}_{32}$. This implies

$$
\left[P_{2}: L_{2}\right]=\left[P_{3}: L_{2}\right]=1, \quad\left[P_{1}: L_{2}\right]=0 .
$$

From $\left[P_{1}: L_{2}\right]=0$ we have $\epsilon_{2} B \epsilon_{1}=0$. This means that

$$
\operatorname{Hom}_{B-B}\left(B \epsilon_{3} \otimes \epsilon_{1} B, B \epsilon_{3} \otimes \epsilon_{2} B\right)=0,
$$

that is, $\operatorname{Hom}\left(\mathrm{G}_{31}, \mathrm{G}_{32}\right)=0$. This contradicts $\operatorname{Hom}_{\mathscr{C}}\left(\mathrm{F}_{21}, \mathrm{~F}_{22}\right) \neq 0$, see (7).

Subcase 3.2. Assume that $\mathrm{F}_{22}$ acts via $\mathrm{G}_{33}$. This implies

$$
\left[P_{3}: L_{3}\right]=\left[P_{2}: L_{3}\right]=1, \quad\left[P_{1}: L_{3}\right]=0 .
$$

From $\left[P_{1}: L_{3}\right]=0$ we have $\epsilon_{3} B \epsilon_{1}=0$. This means that

$$
\operatorname{Hom}_{B-B}\left(B \epsilon_{3} \otimes \epsilon_{1} B, B \epsilon_{3} \otimes \epsilon_{3} B\right)=0,
$$

that is, $\operatorname{Hom}\left(\mathrm{G}_{31}, \mathrm{G}_{33}\right)=0$. This contradicts $\operatorname{Hom}_{\mathscr{C}}\left(\mathrm{F}_{21}, \mathrm{~F}_{22}\right) \neq 0$, see (7). The proof is now complete.

$$
\text { 7. The ALGEBRA } \mathbb{k}(\bullet \stackrel{\alpha}{\rightarrow} \bullet \stackrel{\beta}{\rightarrow} \bullet) /(\beta \alpha)
$$

Let $\mathbb{k}$ be an algebraically closed field. Denote by $A$ the path algebra, over $\mathbb{k}$, of the quiver

$$
\mathbb{k}(1 \stackrel{\alpha}{\longrightarrow} 2 \stackrel{\beta}{\longrightarrow} 3) \quad \text { modulo the relations } \beta \alpha=0 .
$$

The algebra $A$ has basis $e_{1}, e_{2}, e_{3}, \alpha$ and $\beta$ and the multiplication table $(x, y) \mapsto x \cdot y$ is given by:

\begin{tabular}{c||c|c|c|c|c}
$x \backslash y$ & $e_{1}$ & $e_{2}$ & $e_{3}$ & $\alpha$ & $\beta$ \\
\hline \hline$e_{1}$ & $e_{1}$ & 0 & 0 & 0 & 0 \\
\hline$e_{2}$ & 0 & $e_{2}$ & 0 & $\alpha$ & 0 \\
\hline$e_{3}$ & 0 & 0 & $e_{3}$ & 0 & $\beta$ \\
\hline$\alpha$ & $\alpha$ & 0 & 0 & 0 & 0 \\
\hline$\beta$ & 0 & $\beta$ & 0 & 0 & 0
\end{tabular}


Note that $e_{1} A e_{2}=0, e_{1} A e_{3}=0, e_{2} A e_{3}=0$ and $e_{3} A e_{1}=0$. Note also that the left $A$-modules $A e_{1}$ and $\operatorname{Hom}_{\mathbb{k}}\left(e_{2} A, \mathbb{k}\right)$ are isomorphic and the left $A$-modules $A e_{2}$ and $\operatorname{Hom}_{\mathbb{k}}\left(e_{3} A, \mathbb{k}\right)$ are isomorphic.

Let $\mathcal{C}$ be a small category equivalent to $A$-mod. Consider the corresponding finitary 2-category $\mathscr{C}_{A}$. Up to isomorphism, indecomposable 1-morphisms in $\mathscr{C}_{A}$ are $\mathrm{F}_{0}$ and $\mathrm{F}_{i j}$, where $i, j=1,2,3$. Note that formula (2) for $A$ reads $\mathrm{F} \circ \mathrm{F} \cong \mathrm{F}^{\oplus 5}$. Using (11), the table of compositions for the functors $\mathrm{F}_{i j}$ (up to isomorphism) is as follows:

\begin{tabular}{c||c|c|c|c|c|c|c|c|c}
$\circ$ & $\mathrm{F}_{11}$ & $\mathrm{~F}_{12}$ & $\mathrm{~F}_{13}$ & $\mathrm{~F}_{21}$ & $\mathrm{~F}_{22}$ & $\mathrm{~F}_{23}$ & $\mathrm{~F}_{31}$ & $\mathrm{~F}_{32}$ & $\mathrm{~F}_{33}$ \\
\hline \hline $\mathrm{F}_{11}$ & $\mathrm{~F}_{11}$ & $\mathrm{~F}_{12}$ & $\mathrm{~F}_{13}$ & 0 & 0 & 0 & 0 & 0 & 0 \\
\hline $\mathrm{F}_{12}$ & $\mathrm{~F}_{11}$ & $\mathrm{~F}_{12}$ & $\mathrm{~F}_{13}$ & $\mathrm{~F}_{11}$ & $\mathrm{~F}_{12}$ & $\mathrm{~F}_{13}$ & 0 & 0 & 0 \\
\hline $\mathrm{F}_{13}$ & 0 & 0 & 0 & $\mathrm{~F}_{11}$ & $\mathrm{~F}_{12}$ & $\mathrm{~F}_{13}$ & $\mathrm{~F}_{11}$ & $\mathrm{~F}_{12}$ & $\mathrm{~F}_{13}$ \\
\hline $\mathrm{F}_{21}$ & $\mathrm{~F}_{21}$ & $\mathrm{~F}_{22}$ & $\mathrm{~F}_{23}$ & 0 & 0 & 0 & 0 & 0 & 0 \\
\hline $\mathrm{F}_{22}$ & $\mathrm{~F}_{21}$ & $\mathrm{~F}_{22}$ & $\mathrm{~F}_{23}$ & $\mathrm{~F}_{21}$ & $\mathrm{~F}_{22}$ & $\mathrm{~F}_{23}$ & 0 & 0 & 0 \\
\hline $\mathrm{F}_{23}$ & 0 & 0 & 0 & $\mathrm{~F}_{21}$ & $\mathrm{~F}_{22}$ & $\mathrm{~F}_{23}$ & $\mathrm{~F}_{21}$ & $\mathrm{~F}_{22}$ & $\mathrm{~F}_{23}$ \\
\hline $\mathrm{F}_{31}$ & $\mathrm{~F}_{31}$ & $\mathrm{~F}_{32}$ & $\mathrm{~F}_{33}$ & 0 & 0 & 0 & 0 & 0 & 0 \\
\hline $\mathrm{F}_{32}$ & $\mathrm{~F}_{31}$ & $\mathrm{~F}_{32}$ & $\mathrm{~F}_{33}$ & $\mathrm{~F}_{31}$ & $\mathrm{~F}_{32}$ & $\mathrm{~F}_{33}$ & 0 & 0 & 0 \\
\hline $\mathrm{F}_{33}$ & 0 & 0 & 0 & $\mathrm{~F}_{31}$ & $\mathrm{~F}_{32}$ & $\mathrm{~F}_{33}$ & $\mathrm{~F}_{31}$ & $\mathrm{~F}_{32}$ & $\mathrm{~F}_{33}$
\end{tabular}

Set $\mathcal{J}_{0}:=\left\{\mathrm{F}_{0}\right\}$ and $\mathcal{J}:=\left\{\mathrm{F}_{i j}: i, j=1,2,3\right\}$. Note that the 2-category $\mathscr{C}_{A}$ is not weakly fiat in the sense of [MM2, MM6] as the algebra $A$ is not self-injective.

As $\mathscr{C}_{A}$ is $\mathcal{J}$-simple and $A$ has trivial center, the only proper non-zero quotient of $\mathscr{C}_{A}$ contains just the identity 1-morphism (up to isomorphism) and its scalar endomorphisms (cf. MM3]). Therefore this quotient is fiat with strongly regular $\mathcal{J}$-classes and hence it has a unique, up to equivalence, simple transitive 2-representation, namely $\mathbf{C}_{\mathcal{L}_{0}}$, where $\mathcal{L}_{0}=\mathcal{J}_{0}$, see [MM5, Theorem 18]. This means that, in order to prove Theorem 1 for $A$, it is enough to consider faithful 2-representations of $\mathscr{C}_{A}$.

From (6), we get the following table of $\operatorname{Hom}_{\mathscr{C}_{A}(\mathrm{i})}(X, Y)$ (up to isomorphism), where $X$ and $Y$ are indecomposable 1-morphisms as specified in the table:

\begin{tabular}{c||c|c|c|c|c|c|c|c|c}
$X \backslash Y$ & $\mathrm{~F}_{11}$ & $\mathrm{~F}_{12}$ & $\mathrm{~F}_{13}$ & $\mathrm{~F}_{21}$ & $\mathrm{~F}_{22}$ & $\mathrm{~F}_{23}$ & $\mathrm{~F}_{31}$ & $\mathrm{~F}_{32}$ & $\mathrm{~F}_{33}$ \\
\hline \hline $\mathrm{F}_{11}$ & $\mathbb{k}$ & $\mathbb{k}$ & 0 & 0 & 0 & 0 & 0 & 0 & 0 \\
\hline $\mathrm{F}_{12}$ & 0 & $\mathbb{k}$ & $\mathbb{k}$ & 0 & 0 & 0 & 0 & 0 & 0 \\
\hline $\mathrm{F}_{13}$ & 0 & 0 & $\mathbb{k}$ & 0 & 0 & 0 & 0 & 0 & 0 \\
\hline $\mathrm{F}_{21}$ & $\mathbb{k}$ & $\mathbb{k}$ & 0 & $\mathbb{k}$ & $\mathbb{k}$ & 0 & 0 & 0 & 0 \\
\hline $\mathrm{F}_{22}$ & 0 & $\mathbb{k}$ & $\mathbb{k}$ & 0 & $\mathbb{k}$ & $\mathbb{k}$ & 0 & 0 & 0 \\
\hline $\mathrm{F}_{23}$ & 0 & 0 & $\mathbb{k}$ & 0 & 0 & $\mathbb{k}$ & 0 & 0 & 0 \\
\hline $\mathrm{F}_{31}$ & 0 & 0 & 0 & $\mathbb{k}$ & $\mathbb{k}$ & 0 & $\mathbb{k}$ & $\mathbb{k}$ & 0 \\
\hline $\mathrm{F}_{32}$ & 0 & 0 & 0 & 0 & $\mathbb{k}$ & $\mathbb{k}$ & 0 & $\mathbb{k}$ & $\mathbb{k}$ \\
\hline $\mathrm{F}_{33}$ & 0 & 0 & 0 & 0 & 0 & $\mathbb{k}$ & 0 & 0 & $\mathbb{k}$
\end{tabular}

\section{INTEGER MATRICES FOR $\mathbb{k}(\bullet \stackrel{\alpha}{\rightarrow} \bullet \stackrel{\beta}{\rightarrow} \bullet) /(\beta \alpha)$}

8.1. Integer matrices satisfying $M^{2}=5 M$. In this section we classify all square matrices $M$ with positive integer coefficients which satisfy $M^{2}=5 M$.

Proposition 12. Let $M$ be $a k \times k$ matrix, for some $k$, with positive integer coefficients, satisfying $M^{2}=5 M$. Then, up to permutation action, $M$ is one of the 
following matrices:

$$
\begin{aligned}
& N_{1}:=(5), \quad N_{2}:=\left(\begin{array}{ll}
4 & 1 \\
4 & 1
\end{array}\right), \quad N_{3}:=\left(\begin{array}{ll}
4 & 4 \\
1 & 1
\end{array}\right), \quad N_{4}:=\left(\begin{array}{ll}
4 & 2 \\
2 & 1
\end{array}\right), \\
& N_{5}:=\left(\begin{array}{cc}
3 & 6 \\
1 & 2
\end{array}\right), \quad N_{6}:=\left(\begin{array}{cc}
3 & 3 \\
2 & 2
\end{array}\right), \quad N_{7}:=\left(\begin{array}{ll}
3 & 2 \\
3 & 2
\end{array}\right), \quad N_{8}:=\left(\begin{array}{ll}
3 & 1 \\
6 & 2
\end{array}\right), \\
& N_{9}:=\left(\begin{array}{ccc}
3 & 1 & 1 \\
3 & 1 & 1 \\
3 & 1 & 1
\end{array}\right), \quad N_{10}:=\left(\begin{array}{ccc}
3 & 3 & 3 \\
1 & 1 & 1 \\
1 & 1 & 1
\end{array}\right), \quad N_{11}:=\left(\begin{array}{lll}
2 & 2 & 2 \\
2 & 2 & 2 \\
1 & 1 & 1
\end{array}\right) \text {, } \\
& N_{12}:=\left(\begin{array}{ccc}
2 & 4 & 2 \\
1 & 2 & 1 \\
1 & 2 & 1
\end{array}\right), \quad N_{13}:=\left(\begin{array}{ccc}
2 & 2 & 1 \\
2 & 2 & 1 \\
2 & 2 & 1
\end{array}\right), \quad N_{14}:=\left(\begin{array}{ccccc}
1 & 1 & 1 & 1 & 1 \\
1 & 1 & 1 & 1 & 1 \\
1 & 1 & 1 & 1 & 1 \\
1 & 1 & 1 & 1 & 1 \\
1 & 1 & 1 & 1 & 1
\end{array}\right) \text {, } \\
& N_{15}:=\left(\begin{array}{cccc}
2 & 1 & 1 & 1 \\
2 & 1 & 1 & 1 \\
2 & 1 & 1 & 1 \\
2 & 1 & 1 & 1
\end{array}\right), \quad N_{16}:=\left(\begin{array}{cccc}
2 & 2 & 2 & 2 \\
1 & 1 & 1 & 1 \\
1 & 1 & 1 & 1 \\
1 & 1 & 1 & 1
\end{array}\right)
\end{aligned}
$$

We note an important difference with Proposition [10 to make our list shorter, Proposition 12 gives classification only up to permutation action.

Proof. Clearly, we have $N_{i}^{2}=5 N_{i}$, for each $i=1,2, \ldots, 16$. So, we need to show that any other square matrix with positive integer coefficients satisfying $M^{2}=5 M$ can be reduced to one of the above using permutation action.

Let $M$ be a $k \times k$ matrix, for some $k$, with positive integer coefficients satisfying $M^{2}=5 M$. Then $M$ is diagonalizable (as $x^{2}-5 x$ has no multiple roots) and the only possible eigenvalues for $M$ are 0 and 5 . From the Perron-Frobenius theorem it follows that the Perron-Frobenius eigenvalue 5 must have multiplicity one. Therefore $M$ has rank one and trace five. As all entries in $M$ are positive integers, we get $k \leq 5$. Using the permutation action, we may assume that the entries on the main diagonal of $M$ weakly decrease from the top left corner to the bottom right corner.

If $k=1$, then, clearly, $M=N_{1}$.

If $k=2$, then the diagonal of $M$ is either $(4,1)$ or $(3,2)$. In the first case, as the determinant of $M$ is zero, the two remaining entries are either 2 and 2 or 4 and 1. This gives $M=N_{2}, M=N_{3}$ or $M=N_{4}$. In the second case, as the determinant of $M$ is zero, the two remaining entries are either 2 and 3 or 1 and 6 . This gives $M=N_{5}, M=N_{6}, M=N_{7}$ or $M=N_{8}$.

If $k=3$, then the diagonal of $M$ is either $(3,1,1)$ or $(2,2,1)$. In the first case, as $M$ has rank one, any $2 \times 2$ minor in $M$ has determinant zero. This means that all entries which are neither in the first row nor in the first column are equal to 1 . If the first row contains more than one entry different from 1, then all entires in this row are 3 and we get $M=N_{10}$. If the first column contains more than one entry different from 1, then all entires in this column are 3 and we get $M=N_{9}$.

In the second case, write

$$
M=\left(\begin{array}{ccc}
2 & m_{12} & m_{13} \\
m_{21} & 2 & m_{23} \\
m_{31} & m_{32} & 1
\end{array}\right)
$$


Then $m_{32} m_{23}=2, m_{31} m_{13}=2$ and $m_{21} m_{12}=4$. Hence, both $\left(m_{32}, m_{23}\right)$ and $\left(m_{31}, m_{13}\right)$ are in $\{(1,2),(2,1)\}$. We can choose them independently and the fact that $M$ has rank one then uniquely determines the pair $\left(m_{21}, m_{12}\right)$. This gives us $M=N_{11}, M=N_{12}$ and $M=N_{13}$ and also the possibility

$$
M=N_{12}^{\prime}:=\left(\begin{array}{ccc}
2 & 1 & 1 \\
4 & 2 & 2 \\
2 & 1 & 1
\end{array}\right) .
$$

which reduces to $M=N_{12}$ by permutation action.

If $k=4$, then the diagonal of $M$ is $(2,1,1,1)$. As $M$ has rank one, any $2 \times 2$ minor in $M$ has determinant zero. This means that all entries which are neither in the first row nor in the first column are equal to 1 . If the first row contains more than one entry different from 1 , then all entires in this row are 2 and we get $M=N_{16}$. If the first column contains more than one entry different from 1 , then all entires in this column are 2 and we get $M=N_{15}$.

If $k=5$, then all diagonal entries in $M$ are 1 . As all $2 \times 2$ minors in $M$ should have determinant zero and positive integer entries, it follows that all entries in $M$ are 1 and thus $M=N_{14}$.

8.2. Filtering "easy cases" out. Let $\mathbf{M}$ be a finitary, simple, transitive and faithful 2-representation of $\mathscr{C}_{A}$. Let $M:=[\mathrm{F}]$ be the matrix of $\mathbf{M}(\mathrm{F})$ and, for $i, j=1,2,3$, let $M_{i j}:=\left[\mathrm{F}_{i j}\right]$ be the matrix of $\mathbf{M}\left(\mathrm{F}_{i j}\right)$. We have $M_{i j} \neq 0$, for all $i, j=1,2,3$. By Proposition [12, up to permutation action, we have $M=N_{i}$, for some $i \in\{1,2, \ldots, 16\}$ as in Proposition 12, Note that trace of $M$ is five.

As usual, we call "position $(i, j)$ " the intersection of the $i$-th row and the $j$-th column of a matrix.

From now on, we assume that $\overline{\mathbf{M}}(i)$ is equivalent to $B$-mod, for some basic algebra $B$. Let $L_{1}, L_{2}, \ldots, L_{k}$ be a complete and irredundant list of representatives of isomorphism classes of simple objects in $\overline{\mathbf{M}}(\mathrm{i})$. For $i \in\{1,2, \ldots, k\}$, denote by $P_{i}$ the indecomposable projective cover of $L_{i}$ and by $I_{i}$ the indecomposable injective envelope of $L_{i}$. The matrices $M_{i j}$ are given with respect to this fixed ordering of isomorphism classes of simple objects.

\section{Lemma 13.}

(i) All diagonal elements in $M_{13}, M_{21}, M_{31}$ and $M_{32}$ are zero.

(ii) Each of the matrices $M_{11}, M_{12}, M_{22}, M_{23}$ and $M_{33}$, has exactly one entry equal to 1 on the diagonal and all other diagonal entries are zero.

Proof. From (8), we see that $\mathrm{F}_{i j}$ is idempotent if and only if

$$
(i, j) \in\{(1,1),(1,2),(2,2),(2,3),(3,3)\}
$$

and $\mathrm{F}_{i j}^{2}=0$ otherwise. As the trace of a non-zero idempotent with non-negative coefficients is non-zero, each idempotent $M_{i j}$ has trace at least one. As trace of $M$ is five, it follows that all idempotents $M_{i j}$ have trace one. This proves claim (iii). Claim (ii) follows from claim (iii) as $M$ is the sum of the $M_{i j}$ 's.

Corollary 14. The matrix $M$ cannot be equal to $N_{i}$, where $i \in\{1,2, \ldots, 10\}$.

Proof. Each of the matrices $N_{i}$, where $i \in\{1,2, \ldots, 10\}$, contains a diagonal element which is greater than or equal to 3 . If $M=N_{i}$ would be possible, at least three idempotents $M_{i j}$ would have this diagonal element non-zero. But then any 
product of any two such matrices would be non-zero. However, from (8) we have that, for any three different idempotents $\mathrm{F}_{i j}$, one of the products of two of these elements is zero. The obtained contradiction completes the proof.

8.3. Auxiliary adjunction. We will need the following easy observations:

Lemma 15. Let $D$ be a finite dimensional algebra and $(\mathrm{G}, \mathrm{H})$ an adjoint pair of right exact endofunctors of D-mod. Let $L$ and $L^{\prime}$ be simple $D$-modules and $P$ and $P^{\prime}$ their corresponding indecomposable projective covers. Assume that $L^{\prime}$ appears in the top of $\mathrm{G} P$. Then $\mathrm{H} P^{\prime} \neq 0$.

Proof. By adjunction, we have

$$
0 \neq \operatorname{Hom}_{B}\left(\mathrm{G} P, L^{\prime}\right) \cong \operatorname{Hom}_{B}\left(P, \mathrm{H} L^{\prime}\right),
$$

which implies $\mathrm{H} L^{\prime} \neq 0$. As $\mathrm{H}$ is right exact, this forces $\mathrm{H} P^{\prime} \neq 0$.

Lemma 16. We have the following pairs of adjoint 1-morphisms in $\mathscr{C}_{A}$ :

$$
\left(\mathrm{F}_{33}, \mathrm{~F}_{23}\right), \quad\left(\mathrm{F}_{23}, \mathrm{~F}_{22}\right), \quad\left(\mathrm{F}_{22}, \mathrm{~F}_{12}\right), \quad\left(\mathrm{F}_{12}, \mathrm{~F}_{11}\right) \text {. }
$$

Proof. As both, the left $A$-modules $A e_{1}$ and $\operatorname{Hom}_{\mathbb{k}}\left(e_{2} A, \mathbb{k}\right)$ are isomorphic and the left $A$-modules $A e_{2}$ and $\operatorname{Hom}_{\mathbb{k}}\left(e_{3} A, \mathbb{k}\right)$ are isomorphic, the claim follows from Lemma 5 .

8.4. Idempotent integral matrices of rank one. Recall from [Fl, Theorem 2] that, up to permutation action, idempotent matrices of rank one with non-negative integer entries have the form

$$
\left(\begin{array}{ccc}
\mathbf{0} & v & v w^{t} \\
\mathbf{0} & 1 & w^{t} \\
\mathbf{0} & \mathbf{0} & \mathbf{0}
\end{array}\right)
$$

where $\mathbf{0}$ denotes the zero matrix (of an appropriate size), and $v$ and $w$ are arbitrary vectors with non-negative integer entries. In particular, if the diagonal entry 1 is in the $i$-th row, then the whole matrix can be written as the product of its $i$-th column with its $i$-th row.

\subsection{Filtering matrices $N_{14}, N_{15}$ and $N_{16}$ out.}

Proposition 17. The matrix $M$ cannot be equal to $N_{14}, N_{15}$ or $N_{16}$.

Proof. Assume that the diagonals of the matrices $M_{11}$ and $M_{12}$ are different. This means that $M_{11}$ has 1 in row $i$, that $M_{12}$ has 1 in row $j$, and that $i \neq j$.

Then $\mathrm{F}_{12} P_{j}$ has $P_{j}$ as a direct summand. Therefore, by combining Lemmata 15 and 16. we have that $M_{11}$ must have a non-zero element in column $j$. From Subsection 8.4 it follows that $M_{11}$ has a non-zero entry in position $(i, j)$.

As $M_{11}$ has a non-zero entry in position $(i, j)$ and the matrix $M_{12}$ has 1 in position $(j, j)$, it follows that $M_{11} M_{12}$ has a non-zero entry in position $(i, j)$. From (8), we have $M_{11} M_{12}=M_{12}$, which means that $M_{12}$ has a non-zero entry in position $(i, j)$. This already means that the case $M=N_{14}$ is not possible.

Assume $M=N_{15}$. Then we must have $j=1$. Exactly the same argument as above applied to $M_{22}$ and $M_{23}$ shows that $M_{23}$ has 1 in position $(1,1)$. This contradicts $M_{23} M_{12}=0$ as follows from (8). Therefore $M=N_{15}$ is not possible. 
Assume $M=N_{16}$. Then we must have $i=1$. Exactly the same argument as above applied to $M_{22}$ and $M_{23}$ shows that $M_{22}$ has 1 in position $(1,1)$. This contradicts $M_{11} M_{22}=0$ as follows from (8). Therefore $M=N_{16}$ is not possible. This completes the proof.

The remaining cases for $M$ will be studied on a case-by-case basis.

\section{Filtering matrices $N_{11}$ And $N_{12}$ OUt}

9.1. Statement. The main aim of this section is to prove the following

Proposition 18. The matrix $M$ cannot be equal to $N_{11}$ or $N_{12}$.

We start with the following observation.

Lemma 19. The only unordered pairs of idempotent 1-morphisms of the form $\mathrm{F}_{i j}$ such that the product of any two elements in the pair is non-zero are

$$
\left\{\mathrm{F}_{11}, \mathrm{~F}_{12}\right\}, \quad\left\{\mathrm{F}_{12}, \mathrm{~F}_{22}\right\}, \quad\left\{\mathrm{F}_{22}, \mathrm{~F}_{23}\right\}, \quad\left\{\mathrm{F}_{23}, \mathrm{~F}_{33}\right\} .
$$

Proof. This follows directly from (8).

9.2. Proof for $M=N_{11}$. We will arrange matrices $M_{i j}$, where $i, j=1,2,3$, as follows:

$$
\begin{array}{lll}
M_{11} & M_{12} & M_{13} \\
M_{21} & M_{22} & M_{23} \\
M_{31} & M_{32} & M_{33}
\end{array}
$$

Assume $M=N_{11}$. The diagonal elements in $N_{11}$ are $(2,2,1)$. Therefore, two pairs of idempotent matrices of the form $M_{i j}$ would have common diagonal elements. Any product of matrices in any such pair would be non-zero. Therefore, using Lemma 19, we have three cases to consider.

Case 1. Suppose first that the pairs of idempotent matrices which share diagonal elements are $\left\{\mathrm{F}_{11}, \mathrm{~F}_{12}\right\}$ and $\left\{\mathrm{F}_{22}, \mathrm{~F}_{23}\right\}$. Up to permutation action, we may assume that

$$
\begin{aligned}
& \left(\begin{array}{lll}
1 & * & * \\
* & 0 & * \\
* & * & 0
\end{array}\right), \quad\left(\begin{array}{lll}
1 & * & * \\
* & 0 & * \\
* & * & 0
\end{array}\right), \quad\left(\begin{array}{lll}
0 & * & * \\
* & 0 & * \\
* & * & 0
\end{array}\right), \\
& \left(\begin{array}{lll}
0 & * & * \\
* & 0 & * \\
* & * & 0
\end{array}\right), \quad\left(\begin{array}{lll}
0 & * & * \\
* & 1 & * \\
* & * & 0
\end{array}\right), \quad\left(\begin{array}{lll}
0 & * & * \\
* & 1 & * \\
* & * & 0
\end{array}\right), \\
& \left(\begin{array}{lll}
0 & * & * \\
* & 0 & * \\
* & * & 0
\end{array}\right), \quad\left(\begin{array}{lll}
0 & * & * \\
* & 0 & * \\
* & * & 0
\end{array}\right), \quad\left(\begin{array}{lll}
0 & * & * \\
* & 0 & * \\
* & * & 1
\end{array}\right) .
\end{aligned}
$$

Using all possible zero products which appear in (8), we obtain that the $M_{i j}$ 's look as follows:

$$
\begin{aligned}
& \left(\begin{array}{lll}
1 & 0 & 0 \\
0 & 0 & 0 \\
0 & 0 & 0
\end{array}\right), \quad\left(\begin{array}{lll}
1 & * & 0 \\
0 & 0 & 0 \\
0 & 0 & 0
\end{array}\right), \quad\left(\begin{array}{lll}
0 & * & * \\
0 & 0 & 0 \\
0 & 0 & 0
\end{array}\right), \\
& \left(\begin{array}{lll}
0 & 0 & 0 \\
* & 0 & 0 \\
* & 0 & 0
\end{array}\right), \quad\left(\begin{array}{lll}
0 & 0 & 0 \\
* & 1 & 0 \\
* & * & 0
\end{array}\right), \quad\left(\begin{array}{lll}
0 & 0 & 0 \\
0 & 1 & * \\
0 & * & 0
\end{array}\right),
\end{aligned}
$$




$$
\left(\begin{array}{lll}
0 & 0 & 0 \\
0 & 0 & 0 \\
* & 0 & 0
\end{array}\right), \quad\left(\begin{array}{lll}
0 & 0 & 0 \\
0 & 0 & 0 \\
* & * & 0
\end{array}\right), \quad\left(\begin{array}{lll}
0 & 0 & 0 \\
0 & 0 & 0 \\
0 & * & 1
\end{array}\right) .
$$

As all matrices must be non-zero and add up to $M$, we obtain

$$
\begin{aligned}
& \left(\begin{array}{lll}
1 & 0 & 0 \\
0 & 0 & 0 \\
0 & 0 & 0
\end{array}\right), \quad\left(\begin{array}{lll}
1 & * & 0 \\
0 & 0 & 0 \\
0 & 0 & 0
\end{array}\right), \quad\left(\begin{array}{lll}
0 & * & 2 \\
0 & 0 & 0 \\
0 & 0 & 0
\end{array}\right), \\
& \left(\begin{array}{lll}
0 & 0 & 0 \\
* & 0 & 0 \\
0 & 0 & 0
\end{array}\right), \quad\left(\begin{array}{lll}
0 & 0 & 0 \\
* & 1 & 0 \\
0 & 0 & 0
\end{array}\right), \quad\left(\begin{array}{lll}
0 & 0 & 0 \\
0 & 1 & 2 \\
0 & 0 & 0
\end{array}\right), \\
& \left(\begin{array}{lll}
0 & 0 & 0 \\
0 & 0 & 0 \\
1 & 0 & 0
\end{array}\right), \quad\left(\begin{array}{lll}
0 & 0 & 0 \\
0 & 0 & 0 \\
0 & 1 & 0
\end{array}\right), \quad\left(\begin{array}{lll}
0 & 0 & 0 \\
0 & 0 & 0 \\
0 & 0 & 1
\end{array}\right) .
\end{aligned}
$$

This contradicts $M_{13} M_{31}=M_{11}$ which is a consequence of (8). Therefore this case is not possible.

Case 2. Suppose now that the pairs of idempotent matrices which share diagonal elements are $\left\{\mathrm{F}_{12}, \mathrm{~F}_{22}\right\}$ and $\left\{\mathrm{F}_{23}, \mathrm{~F}_{33}\right\}$. Up to permutation action, we may assume that the $M_{i j}$ 's look as follows:

$$
\begin{aligned}
& \left(\begin{array}{lll}
0 & * & * \\
* & 0 & * \\
* & * & 1
\end{array}\right), \quad\left(\begin{array}{lll}
1 & * & * \\
* & 0 & * \\
* & * & 0
\end{array}\right), \quad\left(\begin{array}{lll}
0 & * & * \\
* & 0 & * \\
* & * & 0
\end{array}\right), \\
& \left(\begin{array}{lll}
0 & * & * \\
* & 0 & * \\
* & * & 0
\end{array}\right), \quad\left(\begin{array}{lll}
1 & * & * \\
* & 0 & * \\
* & * & 0
\end{array}\right), \quad\left(\begin{array}{lll}
0 & * & * \\
* & 1 & * \\
* & * & 0
\end{array}\right), \\
& \left(\begin{array}{lll}
0 & * & * \\
* & 0 & * \\
* & * & 0
\end{array}\right), \quad\left(\begin{array}{lll}
0 & * & * \\
* & 0 & * \\
* & * & 0
\end{array}\right), \quad\left(\begin{array}{lll}
0 & * & * \\
* & 1 & * \\
* & * & 0
\end{array}\right) .
\end{aligned}
$$

Using all possible zero products which appear in (8), we obtain that the $M_{i j}$ 's look as follows:

$$
\begin{aligned}
& \left(\begin{array}{lll}
0 & 0 & * \\
0 & 0 & 0 \\
0 & 0 & 1
\end{array}\right), \quad\left(\begin{array}{lll}
1 & 0 & * \\
0 & 0 & 0 \\
* & 0 & 0
\end{array}\right), \quad\left(\begin{array}{lll}
0 & * & 0 \\
0 & 0 & 0 \\
0 & * & 0
\end{array}\right), \\
& \left(\begin{array}{lll}
0 & 0 & * \\
0 & 0 & * \\
0 & 0 & 0
\end{array}\right), \quad\left(\begin{array}{lll}
1 & 0 & * \\
* & 0 & * \\
0 & 0 & 0
\end{array}\right), \quad\left(\begin{array}{lll}
0 & * & 0 \\
0 & 1 & 0 \\
0 & 0 & 0
\end{array}\right), \\
& \left(\begin{array}{lll}
0 & 0 & 0 \\
0 & 0 & * \\
0 & 0 & 0
\end{array}\right), \quad\left(\begin{array}{lll}
0 & 0 & 0 \\
* & 0 & * \\
0 & 0 & 0
\end{array}\right), \quad\left(\begin{array}{lll}
0 & 0 & 0 \\
0 & 1 & 0 \\
0 & 0 & 0
\end{array}\right) .
\end{aligned}
$$

Here we have that $P_{2}$ is a direct summand of $\mathrm{F}_{23} P_{2}$. From Lemmata 15 and 16 , it follows that $\mathrm{F}_{22} P_{2} \neq 0$, which is a contradiction. Therefore this case cannot occur either.

Case 3. Suppose first that the pairs of idempotent matrices which share diagonal elements are $\left\{\mathrm{F}_{11}, \mathrm{~F}_{12}\right\}$ and $\left\{\mathrm{F}_{23}, \mathrm{~F}_{33}\right\}$. Up to permutation action, we may assume that the $M_{i j}$ 's look as follows:

$$
\left(\begin{array}{lll}
1 & * & * \\
* & 0 & * \\
* & * & 0
\end{array}\right), \quad\left(\begin{array}{lll}
1 & * & * \\
* & 0 & * \\
* & * & 0
\end{array}\right), \quad\left(\begin{array}{lll}
0 & * & * \\
* & 0 & * \\
* & * & 0
\end{array}\right),
$$




$$
\begin{aligned}
& \left(\begin{array}{lll}
0 & * & * \\
* & 0 & * \\
* & * & 0
\end{array}\right), \quad\left(\begin{array}{lll}
0 & * & * \\
* & 0 & * \\
* & * & 1
\end{array}\right), \quad\left(\begin{array}{lll}
0 & * & * \\
* & 1 & * \\
* & * & 0
\end{array}\right), \\
& \left(\begin{array}{lll}
0 & * & * \\
* & 0 & * \\
* & * & 0
\end{array}\right), \quad\left(\begin{array}{lll}
0 & * & * \\
* & 0 & * \\
* & * & 0
\end{array}\right), \quad\left(\begin{array}{lll}
0 & * & * \\
* & 1 & * \\
* & * & 0
\end{array}\right) .
\end{aligned}
$$

Using all possible zero products which appear in (8), we obtain that the $M_{i j}$ 's look as follows:

$$
\begin{aligned}
& \left(\begin{array}{lll}
1 & 0 & 0 \\
0 & 0 & 0 \\
* & 0 & 0
\end{array}\right), \quad\left(\begin{array}{lll}
1 & 0 & * \\
0 & 0 & 0 \\
* & 0 & 0
\end{array}\right), \quad\left(\begin{array}{lll}
0 & * & * \\
0 & 0 & 0 \\
0 & * & 0
\end{array}\right), \\
& \left(\begin{array}{ccc}
0 & 0 & 0 \\
* & 0 & 0 \\
* & 0 & 0
\end{array}\right), \quad\left(\begin{array}{ccc}
0 & 0 & 0 \\
* & 0 & * \\
* & 0 & 1
\end{array}\right), \quad\left(\begin{array}{lll}
0 & 0 & 0 \\
0 & 1 & * \\
0 & * & 0
\end{array}\right), \\
& \left(\begin{array}{lll}
0 & 0 & 0 \\
* & 0 & 0 \\
0 & 0 & 0
\end{array}\right), \quad\left(\begin{array}{ccc}
0 & 0 & 0 \\
* & 0 & * \\
0 & 0 & 0
\end{array}\right), \quad\left(\begin{array}{lll}
0 & 0 & 0 \\
0 & 1 & * \\
0 & 0 & 0
\end{array}\right) \text {. }
\end{aligned}
$$

Here we have that $P_{2}$ is a direct summand of $\mathrm{F}_{23} P_{2}$. From Lemmata 15] and 16, it follows that $\mathrm{F}_{22} P_{2} \neq 0$, which is a contradiction. Therefore this case cannot occur either.

This completes the proof of Lemma 19, for $M=N_{11}$.

9.3. Proof for $M=N_{12}$. Let

$$
N_{12}^{\prime}:=\left(\begin{array}{ccc}
2 & 1 & 1 \\
4 & 2 & 2 \\
2 & 1 & 1
\end{array}\right)
$$

The matrix $N_{12}^{\prime}$ reduces to $M=N_{12}$ by permutation action, however, it is convenient to use the freedom of permutation action in another way, see below. Because of Lemma 19, we have three cases to consider.

Case 1. Suppose first that the pairs of idempotent matrices which share diagonal elements are $\left\{\mathrm{F}_{11}, \mathrm{~F}_{12}\right\}$ and $\left\{\mathrm{F}_{22}, \mathrm{~F}_{23}\right\}$. Then, using permutation action and all possible zero products which appear in (8), we obtain that the $M_{i j}$ 's look as follows:

$$
\begin{aligned}
& \left(\begin{array}{lll}
1 & 0 & 0 \\
0 & 0 & 0 \\
0 & 0 & 0
\end{array}\right), \quad\left(\begin{array}{lll}
1 & * & 0 \\
0 & 0 & 0 \\
0 & 0 & 0
\end{array}\right), \quad\left(\begin{array}{lll}
0 & * & * \\
0 & 0 & 0 \\
0 & 0 & 0
\end{array}\right), \\
& \left(\begin{array}{lll}
0 & 0 & 0 \\
* & 0 & 0 \\
* & 0 & 0
\end{array}\right), \quad\left(\begin{array}{lll}
0 & 0 & 0 \\
* & 1 & 0 \\
* & * & 0
\end{array}\right), \quad\left(\begin{array}{lll}
0 & 0 & 0 \\
0 & 1 & * \\
0 & * & 0
\end{array}\right), \\
& \left(\begin{array}{lll}
0 & 0 & 0 \\
0 & 0 & 0 \\
* & 0 & 0
\end{array}\right), \quad\left(\begin{array}{lll}
0 & 0 & 0 \\
0 & 0 & 0 \\
* & * & 0
\end{array}\right), \quad\left(\begin{array}{lll}
0 & 0 & 0 \\
0 & 0 & 0 \\
0 & * & 1
\end{array}\right) .
\end{aligned}
$$

If $M=N_{12}$, then the $(1,3)$-entry of $M_{13}$ equals 2 while the $(3,1)$-entry of $M_{31}$ equals 1 . As the $(1,1)$-entry of $M_{11}$ is 1 , we get a contradiction to $M_{13} M_{31}=M_{11}$, which follows from (8). This implies that $M=N_{12}^{\prime}$ and, using also $M_{12} M_{21}=M_{11}$, we have:

$$
\left(\begin{array}{lll}
1 & 0 & 0 \\
0 & 0 & 0 \\
0 & 0 & 0
\end{array}\right), \quad\left(\begin{array}{lll}
1 & 1 & 0 \\
0 & 0 & 0 \\
0 & 0 & 0
\end{array}\right), \quad\left(\begin{array}{lll}
0 & 0 & 1 \\
0 & 0 & 0 \\
0 & 0 & 0
\end{array}\right),
$$




$$
\begin{aligned}
& \left(\begin{array}{lll}
0 & 0 & 0 \\
1 & 0 & 0 \\
* & 0 & 0
\end{array}\right), \quad\left(\begin{array}{lll}
0 & 0 & 0 \\
* & 1 & 0 \\
* & * & 0
\end{array}\right), \quad\left(\begin{array}{lll}
0 & 0 & 0 \\
0 & 1 & * \\
0 & * & 0
\end{array}\right), \\
& \left(\begin{array}{lll}
0 & 0 & 0 \\
0 & 0 & 0 \\
1 & 0 & 0
\end{array}\right), \quad\left(\begin{array}{lll}
0 & 0 & 0 \\
0 & 0 & 0 \\
* & * & 0
\end{array}\right), \quad\left(\begin{array}{lll}
0 & 0 & 0 \\
0 & 0 & 0 \\
0 & * & 1
\end{array}\right) \text {. }
\end{aligned}
$$

As the $M_{i j}$ 's must add up to $M$, we have

$$
\begin{aligned}
& \left(\begin{array}{lll}
1 & 0 & 0 \\
0 & 0 & 0 \\
0 & 0 & 0
\end{array}\right), \quad\left(\begin{array}{lll}
1 & 1 & 0 \\
0 & 0 & 0 \\
0 & 0 & 0
\end{array}\right), \quad\left(\begin{array}{lll}
0 & 0 & 1 \\
0 & 0 & 0 \\
0 & 0 & 0
\end{array}\right), \\
& \left(\begin{array}{lll}
0 & 0 & 0 \\
1 & 0 & 0 \\
* & 0 & 0
\end{array}\right), \quad\left(\begin{array}{lll}
0 & 0 & 0 \\
3 & 1 & 0 \\
* & * & 0
\end{array}\right), \quad\left(\begin{array}{lll}
0 & 0 & 0 \\
0 & 1 & 2 \\
0 & * & 0
\end{array}\right), \\
& \left(\begin{array}{lll}
0 & 0 & 0 \\
0 & 0 & 0 \\
1 & 0 & 0
\end{array}\right), \quad\left(\begin{array}{lll}
0 & 0 & 0 \\
0 & 0 & 0 \\
* & * & 0
\end{array}\right), \quad\left(\begin{array}{lll}
0 & 0 & 0 \\
0 & 0 & 0 \\
0 & * & 1
\end{array}\right) \text {. }
\end{aligned}
$$

This contradicts $M_{23} M_{31}=M_{21}$, which follows from (8). Therefore this case cannot occur.

Case 2. Suppose now that the pairs of idempotent matrices which share diagonal elements are $\left\{\mathrm{F}_{12}, \mathrm{~F}_{22}\right\}$ and $\left\{\mathrm{F}_{23}, \mathrm{~F}_{33}\right\}$. This gives the same contradiction as in Case 2 in Subsection 9.2, Therefore this case cannot occur either.

Case 3. Suppose first that the pairs of idempotent matrices which share diagonal elements are $\left\{\mathrm{F}_{11}, \mathrm{~F}_{12}\right\}$ and $\left\{\mathrm{F}_{23}, \mathrm{~F}_{33}\right\}$. This gives the same contradiction as in Case 3 in Subsection 9.2. Therefore this case cannot occur either. This completes the proof of Lemma 19

\section{Proof OF Theorem 1 For $\mathbb{k}(\bullet \stackrel{\alpha}{\rightarrow} \bullet \stackrel{\beta}{\rightarrow} \bullet) /(\beta \alpha)$}

10.1. Finding the matrices. Combining Proposition 12 with Corollary 14 Proposition 17 and Proposition 18, we have $M=N_{13}$. We will arrange our matrices similarly to (10).

We will need the following easy and general observation:

Lemma 20. Let $M$ be any of the $N_{m}$ 's and $i, j \in\{1,2,3\}$. If, for some $s$, the column $s$ in the matrix $M_{i j}$ is non-zero, then the column $s$ is non-zero in $M_{t j}$, for any $t \in\{1,2,3\}$.

Proof. The fact that the column $s$ in $M_{i j}$ is non-zero is equivalent to saying that $\mathrm{F}_{i j} P_{s} \neq 0$ (and similarly for $\mathrm{F}_{t j}$ ). We have $\mathrm{F}_{i t} \circ \mathrm{F}_{t j} \cong \mathrm{F}_{i j}$ from (8). Therefore $\mathrm{F}_{t j} P_{s}=0$ implies $\mathrm{F}_{i j} P_{s}=0$ and the claim follows.

Proposition 21. The only possibility for the $M_{i j}$ 's is

$$
\begin{aligned}
& \left(\begin{array}{lll}
1 & 0 & 0 \\
0 & 0 & 0 \\
0 & 0 & 0
\end{array}\right), \quad\left(\begin{array}{lll}
1 & 1 & 0 \\
0 & 0 & 0 \\
0 & 0 & 0
\end{array}\right), \\
& \left(\begin{array}{lll}
0 & 0 & 0 \\
1 & 0 & 0 \\
0 & 0 & 0
\end{array}\right), \quad\left(\begin{array}{lll}
0 & 0 & 0 \\
1 & 1 & 0 \\
0 & 0 & 0
\end{array}\right), \quad\left(\begin{array}{lll}
0 & 1 & 1 \\
0 & 0 & 0 \\
0 & 0 & 0
\end{array}\right), \\
& \left.0 \begin{array}{lll}
0 & 0 & 0 \\
0 & 1 & 1 \\
0 & 0 & 0
\end{array}\right),
\end{aligned}
$$




$$
\left(\begin{array}{lll}
0 & 0 & 0 \\
0 & 0 & 0 \\
1 & 0 & 0
\end{array}\right), \quad\left(\begin{array}{lll}
0 & 0 & 0 \\
0 & 0 & 0 \\
1 & 1 & 0
\end{array}\right), \quad\left(\begin{array}{lll}
0 & 0 & 0 \\
0 & 0 & 0 \\
0 & 1 & 1
\end{array}\right) .
$$

Proof. Due to Lemma 19, we have to consider three cases.

Case 1. Suppose that the pairs of idempotent matrices which share diagonal elements are $\left\{\mathrm{F}_{12}, \mathrm{~F}_{22}\right\}$ and $\left\{\mathrm{F}_{23}, \mathrm{~F}_{33}\right\}$. This gives the same contradiction as in Case 2 in Subsection 9.2. Therefore this case cannot occur.

Case 2. Suppose that the pairs of idempotent matrices which share diagonal elements are $\left\{\mathrm{F}_{11}, \mathrm{~F}_{12}\right\}$ and $\left\{\mathrm{F}_{23}, \mathrm{~F}_{33}\right\}$. This gives the same contradiction as in Case 3 in Subsection 9.2. Therefore this case cannot occur either.

Case 3. Suppose that the pairs of idempotent matrices which share diagonal elements are $\left\{\mathrm{F}_{11}, \mathrm{~F}_{12}\right\}$ and $\left\{\mathrm{F}_{22}, \mathrm{~F}_{23}\right\}$. Then, using permutation action and all possible zero products which appear in (8), we obtain that the $M_{i j}$ 's look as follows:

$$
\begin{aligned}
& \left(\begin{array}{lll}
1 & 0 & 0 \\
0 & 0 & 0 \\
0 & 0 & 0
\end{array}\right), \quad\left(\begin{array}{lll}
1 & * & 0 \\
0 & 0 & 0 \\
0 & 0 & 0
\end{array}\right), \quad\left(\begin{array}{lll}
0 & * & * \\
0 & 0 & 0 \\
0 & 0 & 0
\end{array}\right), \\
& \left(\begin{array}{lll}
0 & 0 & 0 \\
* & 0 & 0 \\
* & 0 & 0
\end{array}\right), \quad\left(\begin{array}{lll}
0 & 0 & 0 \\
* & 1 & 0 \\
* & * & 0
\end{array}\right), \quad\left(\begin{array}{lll}
0 & 0 & 0 \\
0 & 1 & * \\
0 & * & 0
\end{array}\right), \\
& \left(\begin{array}{lll}
0 & 0 & 0 \\
0 & 0 & 0 \\
* & 0 & 0
\end{array}\right), \quad\left(\begin{array}{lll}
0 & 0 & 0 \\
0 & 0 & 0 \\
* & * & 0
\end{array}\right), \quad\left(\begin{array}{lll}
0 & 0 & 0 \\
0 & 0 & 0 \\
0 & * & 1
\end{array}\right) .
\end{aligned}
$$

Using that all matrices must be non-zero and add up to $M$ and also $M_{13} M_{31}=M_{11}$, $M_{21} M_{12}=M_{22}$ and $M_{21} M_{13}=M_{23}$, given by (8), we have

$$
\begin{aligned}
& \left(\begin{array}{lll}
1 & 0 & 0 \\
0 & 0 & 0 \\
0 & 0 & 0
\end{array}\right), \quad\left(\begin{array}{lll}
1 & 1 & 0 \\
0 & 0 & 0 \\
0 & 0 & 0
\end{array}\right), \quad\left(\begin{array}{lll}
0 & 1 & 1 \\
0 & 0 & 0 \\
0 & 0 & 0
\end{array}\right), \\
& \left(\begin{array}{lll}
0 & 0 & 0 \\
1 & 0 & 0 \\
* & 0 & 0
\end{array}\right), \quad\left(\begin{array}{lll}
0 & 0 & 0 \\
1 & 1 & 0 \\
* & * & 0
\end{array}\right), \quad\left(\begin{array}{lll}
0 & 0 & 0 \\
0 & 1 & 1 \\
0 & * & 0
\end{array}\right), \\
& \left(\begin{array}{lll}
0 & 0 & 0 \\
0 & 0 & 0 \\
1 & 0 & 0
\end{array}\right), \quad\left(\begin{array}{lll}
0 & 0 & 0 \\
0 & 0 & 0 \\
* & * & 0
\end{array}\right), \quad\left(\begin{array}{lll}
0 & 0 & 0 \\
0 & 0 & 0 \\
0 & * & 1
\end{array}\right) .
\end{aligned}
$$

Comparing the first and the second columns in $M_{32}$ with those of $M_{22}$ and also the third column in $M_{33}$ with that of $M_{23}$ and using Lemma 20 we get exactly the arrangement in the formulation of our proposition. This completes the proof.

10.2. Connecting to the cell 2-representation. Now we know that the $M_{i j}$ 's have the form as specifies in Proposition 21. For $i, j=1,2,3$, we denote by $\mathrm{G}_{i j}$ the corresponding indecomposable projective endofunctor of $\overline{\mathbf{M}}(i)$.

From the form of $M_{i 1}$, where $i=1,2,3$, we see that $\mathrm{F}_{i 1}$ acts via $\mathrm{G}_{i 1}$ (up to isomorphism). Moreover, we also have $\left[P_{i}: L_{1}\right]=\delta_{i, 1}$.

From the form of $M_{12}$, we see that $\mathrm{F}_{12}$ acts via either $\mathrm{G}_{12}$ or $\mathrm{G}_{11}$ or $\mathrm{G}_{12} \oplus \mathrm{G}_{11}$. However, we already know that $\mathrm{G}_{11}$ has matrix $M_{11}$. This leaves us with the only possibilities of $\mathrm{G}_{12}$ or $\mathrm{G}_{12} \oplus \mathrm{G}_{11}$. 
Assume that $\mathrm{F}_{12}$ acts via $\mathrm{G}_{12} \oplus \mathrm{G}_{11}$. Then the matrix of $\mathrm{G}_{12}$ is

$$
\left(\begin{array}{lll}
0 & 1 & 0 \\
0 & 0 & 0 \\
0 & 0 & 0
\end{array}\right) \text {. }
$$

This implies that $\left[P_{i}: L_{2}\right]=\delta_{i, 2}$, for $i=1,2,3$.

According to (9), there is a non-zero 2-morphism $\alpha: \mathrm{F}_{21} \rightarrow \mathrm{F}_{12}$. As $\mathbf{M}$ is faithful, $\overline{\mathbf{M}}(\alpha)$ is non-zero. Evaluation of the latter at

- $P_{3}$ is zero as $P_{3}$ is annihilated by both $\mathrm{F}_{21}$ and $\mathrm{F}_{12}$;

- $P_{2}$ is zero as $P_{2}$ is annihilated by $\mathrm{F}_{21}$;

- $P_{1}$ is zero as $\mathrm{F}_{12} P_{1} \cong P_{1}, \mathrm{~F}_{21} P_{1} \cong P_{2}$ and we also have

$$
\operatorname{Hom}_{\overline{\mathbf{M}}(\mathrm{i})}\left(P_{2}, P_{1}\right)=\left[P_{1}: L_{2}\right]=0,
$$

by the previous paragraph.

Therefore $\overline{\mathbf{M}}(\alpha)$ must be zero, a contradiction. Consequently, $\mathrm{F}_{12}$ acts via $\mathrm{G}_{12}$, which also implies $\left[P_{1}: L_{2}\right]=1$. From this, it follows that $\mathrm{F}_{i 2}$ acts via $\mathrm{G}_{i 2}$, for $i=1,2,3$.

A similar argument shows that $\mathrm{F}_{i 3}$ acts via $\mathrm{G}_{i 3}$, for $i=1,2,3$, and that

$$
\left[P_{2}: L_{3}\right]=\left[P_{3}: L_{3}\right]=1, \quad\left[P_{3}: L_{1}\right]=\left[P_{3}: L_{2}\right]=0 \text {. }
$$

This means that $B \cong A$ and that each $\mathrm{F}_{i j}$ acts via the corresponding $\mathrm{G}_{i j}$. It now follows by the usual arguments, see [MM5, Proposition 9], that $\overline{\mathbf{M}}$ is equivalent to a cell 2-representation of $\mathscr{C}_{A}$. The claim of Theorem 1 for the algebra $\mathbb{k}(\bullet \stackrel{\cdots \cdots \cdots \cdots}{\longrightarrow} \bullet \stackrel{\cdots}{\longrightarrow})$ follows.

\section{REFERENCES}

[Ag] T. Agerholm. Simple 2-representations and classification of categorifications. PhD Thesis, Århus University, Denmark, 2011.

[BFK] J. Bernstein, I. Frenkel, M. Khovanov. A categorification of the Temperley-Lieb algebra and Schur quotients of $U\left(\mathfrak{s l}_{2}\right)$ via projective and Zuckerman functors. Selecta Math. (N.S.) 5 (1999), no. 2, 199-241.

[CR] J. Chuang, R. Rouquier. Derived equivalences for symmetric groups and $\mathfrak{s l}_{2}-$ categorification. Ann. of Math. (2) 167 (2008), no. 1, 245-298.

[Fl] P. Flor. On groups of non-negative matrices. Compositio Math. 21 (1969), 376-382.

[GM1] A. L. Grensing, V. Mazorchuk. Categorification of the Catalan monoid. Semigroup Forum 89 (2014), no. 1, 155-168.

[GM2] A. L. Grensing, V. Mazorchuk. Categorification using dual projection functors. Preprint arXiv:1501.00095

[KiMa] T. Kildetoft, V. Mazorchuk. Parabolic projective functors in type $A$. Preprint arXiv:1506.07008

[KuMa] G. Kudryavtseva, V. Mazorchuk. On multisemigroups. Port. Math. 72 (2015), no. 1 , 47-80

[Le] T. Leinster. Basic bicategories, Preprint arXiv:math/9810017

[Mac] S. Mac Lane. Categories for the Working Mathematician. Springer-Verlag, 1998.

[Maz] V. Mazorchuk. Lectures on algebraic categorification. QGM Master Class Series. European Mathematical Society (EMS), Zurich, 2012

[MM1] V. Mazorchuk, V. Miemietz. Cell 2-representations of finitary 2-categories. Compositio Math 147 (2011), 1519-1545.

[MM2] V. Mazorchuk, V. Miemietz. Additive versus abelian 2-representations of fiat 2-categories. Moscow Math. J. 14 (2014), No.3, 595-615.

[MM3] V. Mazorchuk, V. Miemietz. Endmorphisms of cell 2-representations. Preprint arXiv:1207.6236.

[MM4] V. Mazorchuk, V. Miemietz. Morita theory for finitary 2-categories. Preprint arXiv:1304.4698. To appear in Quantum Topol. 
[MM5] V. Mazorchuk, V. Miemietz. Transitive 2-representations of finitary 2-categories. Preprint arXiv:1404.7589. To appear in Trans. Amer. Math. Soc.

[MM6] V. Mazorchuk, V. Miemietz. Isotypic faithful 2-representations of $\mathcal{J}$-simple fiat 2categories. Preprint arXiv:1408.6102 To appear in Math. Z.

[Ro1] R. Rouquier. Categorification of the braid groups Preprint arXiv:math/0409593.

[Ro2] R. Rouquier. 2-Kac-Moody algebras. Preprint arXiv:0812.5023

[Xa] Q. Xantcha. Gabriel 2-Quivers for Finitary 2-Categories. Preprint arXiv:1310.1586 To appear in J. London Math. Soc.

[Zh1] X. Zhang. Duflo involutions for 2-categories associated to tree quivers. Preprint arXiv:1501.03052 To appear in J. Algebra Appl.

[Zh2] X. Zhang. Simple transitive 2-representations and Drinfeld center for some finitary 2categories. Preprint arXiv:1506.02402

[Zi] J. Zimmermann. Simple transitive 2-representations of Soergel bimodules in type $B_{2}$. Preprint arXiv:1509.01441

V. M: Department of Mathematics, Uppsala University, Box. 480, SE-75106, Uppsala, SWEDEN, email: mazor@math.uu.se

X. Z: Department of Mathematics, East China Normal University, Minhang District, Dong Chuan Road 500, Shanghai, 200241, P. R. CHINA,

email: scropure@126.com 